This is an author version of the contribution published in Journal of Pure and Applied Algebra

The definitive version is available at http://www.sciencedirect.com/science/article/pii/S0022404914002710 doi:10.1016/j.jpaa.2014.09.033 


\title{
Intrinsic algebraic entropy
}

\author{
Dikran Dikranjan Anna Giordano Bruno Suigi Salce Simone Virili
}

\begin{abstract}
The new notion of intrinsic algebraic entropy ent of endomorphisms of Abelian groups is introduced and investigated. The intrinsic algebraic entropy is compared with the algebraic entropy, a well-known numerical invariant introduced in the sixties and recently deeply studied also in its relations to other fields of Mathematics. In particular, it is shown that the intrinsic algebraic entropy and the algebraic entropy coincide on endomorphisms of torsion Abelian groups, and their precise relation is clarified in the torsion-free case. The Addition Theorem and the Uniqueness Theorem are also proved for $\widetilde{\text { ent}}$, in analogy with similar results for the algebraic entropy. Furthermore, a relevant connection of ent to the algebraic entropy of a continuous endomorphism of a locally compact Abelian group $G$ is pointed out; this allows for the computation of the algebraic entropy in case $G$ is totally disconnected.
\end{abstract}

\section{Introduction}

The algebraic entropy ent for endomorphisms of Abelian groups, as sketched in [2] and developed in [25] by Weiss, and more recently in [9], is defined by using trajectories of finite subgroups, and so its natural setting is that of torsion Abelian groups. Peters gave a different definition of algebraic entropy in [16] for automorphisms of Abelian groups using finite subsets instead of finite subgroups; he obtained in this way the new invariant $h$ which is non-trivial also for torsion-free Abelian groups. This notion was extended to endomorphisms of Abelian groups in [6], where it was proved that $h$ coincides with the algebraic entropy ent for endomorphisms of torsion Abelian groups. Imitating [17] and [6], in 24] the definition of algebraic entropy was extended to all continuous endomorphisms of locally compact Abelian (LCA) groups (see 4.1). As noted in [7] also the commutativity of the groups can be removed in all these cases.

In this paper we extend the family of finite subgroups of an Abelian group $G$ by a suitable larger family of subgroups depending on the fixed endomorphism $\phi$ of $G$, deriving a new notion of algebraic entropy of $\phi$ (see Definition 1.2). This suitable notion of subgroup (see Definition 1.1) is inspired by that of inert subgroup introduced by Belyaev in [3] for inner automorphisms of non-commutative groups and investigated in that setting in [4, [18] and [5]. (Recall that a subgroup $H$ of $G$ is inert if $[H: H \cap \phi(H)]$ is finite for every inner automorphism $\phi$ of $G$; Belyaev [3] gives credit to Kegel for coining the term "inert subgroup".)

Following [6], the definition of algebraic entropy $h(\phi)$ of an endomorphism $\phi: G \rightarrow G$ of an Abelian group $G$ makes use of finite subsets $F$ of $G$ and their partial $\phi$-trajectories

$$
T_{n}(\phi, F)=F+\phi(F)+\phi^{2}(F)+\ldots+\phi^{n-1}(F)
$$

This research was partially supported by "Progetti di Eccellenza 2011/12" of Fondazione CARIPARO"

2010 Mathematics Subject Classification. Primary: 20K30, 20K27; Secondary: 20K15, 22B05, 16D10, 37A35, 11R06

Key words and phrases: Abelian groups, algebraic entropy, inert subgroups, Algebraic Yuzvinski Formula, Mahler measure, locally compact Abelian groups. 
for all $n \in \mathbb{N}_{+}$, which are still finite subsets of $G$. Moreover, the $\phi$-trajectory of $F$ is

$$
T(\phi, F)=\bigcup_{n \in \mathbb{N}_{+}} T_{n}(\phi, F) .
$$

If $F$ is a finite subgroup of $G$, then $T(\phi, F)$ is the smallest $\phi$-invariant subgroup of $G$ containing $F$. According to [6], the limit

$$
H(\phi, F)=\lim _{n \rightarrow \infty} \frac{\log \left|T_{n}(\phi, F)\right|}{n}
$$

does exist and is finite, and it is called the algebraic entropy with respect to $F$ of $\phi$. The algebraic entropy of $\phi$ is

$$
h(\phi)=\sup \{H(\phi, F): F \subseteq G \text { finite }\},
$$

while

$$
\operatorname{ent}(\phi)=\sup \{H(\phi, F): F \in \mathcal{F}(G)\},
$$

where $\mathcal{F}(G)$ denotes the family of all finite subgroups of $G$. As previously noted, $h$ and ent coincide when dealing with torsion Abelian groups. Indeed, denoting as usual by $t(G)$ the torsion subgroup of the Abelian group $G$ (that is fully invariant), we have that $\operatorname{ent}(\phi)=\operatorname{ent}\left(\phi \uparrow_{t(G)}\right)$ as every finite subgroup of $G$ is contained in $t(G)$, and $h\left(\phi \uparrow_{t(G)}\right)=\operatorname{ent}\left(\phi \uparrow_{t(G)}\right)$ since every finite subset of $t(G)$ generates a finite subgroup containing it.

According to a basic fact in the framework of the algebraic entropy ent (see [9, Lemma 1.1]), for a finite subgroup $F$ of $G$, the sequence of non-negative integers

$$
\alpha_{n}=\frac{\left|T_{n+1}(\phi, F)\right|}{\left|T_{n}(\phi, F)\right|}
$$

is non-increasing, hence it is stationary. A crucial consequence of this fact is that

$$
H(\phi, F)=\lim _{n \rightarrow \infty} \log \alpha_{n}=\log \alpha,
$$

where $\alpha=\alpha_{n}$ for all $n$ large enough (see [9, Proposition 1.3]).

A natural question arises: can we compute $H(\phi, F)$ using the formula in (1.3), without asking finiteness of the subgroup $F$ ? The idea of using (1.3) instead of 1.1) to compute the algebraic entropy of $\phi$ with respect to $F$ triggered the new notion of entropy, that we call intrinsic algebraic entropy.

Since for all $n \in \mathbb{N}_{+}$and for every subgroup $H$ of $G$ we have

$$
\frac{T_{n+1}(\phi, H)}{T_{n}(\phi, H)} \cong \frac{T_{n+1}(\phi, H) / H}{T_{n}(\phi, H) / H},
$$

in order to get the limit in 1.3 we do not need finiteness of $T_{n}(\phi, H)$ for all $n \in \mathbb{N}_{+}$. What really matters is finiteness of $T_{n}(\phi, H) / H$ for all $n \in \mathbb{N}_{+}$, hence we are led to the following

Definition 1.1. Let $G$ be an Abelian group, $\phi: G \rightarrow G$ an endomorphism. A subgroup $H$ of $G$ is $\phi$-inert if $(H+\phi(H)) / H$ is finite.

Since $T_{2}(\phi, H) / H=(H+\phi(H)) / H$ is finite if and only if $T_{n}(\phi, H) / H$ is finite for all $n \in \mathbb{N}_{+}$(see Lemma 2.1), in view of (1.4) the limit in (1.3) exists and is finite for a $\phi$-inert subgroup $H$ of $G$ (see Lemma 3.2 ; hence we can set

$$
\widetilde{\operatorname{ent}}(\phi, H)=\lim _{n \rightarrow \infty} \frac{\log \left|T_{n}(\phi, H) / H\right|}{n} .
$$

Taking the supremum of these non-negative real numbers, ranging $H$ in the set $\mathcal{I}_{\phi}(G)$ of the $\phi$-inert subgroups of $G$, we have the announced new notion: 
Definition 1.2. Let $G$ be an Abelian group and $\phi: G \rightarrow G$ an endomorphism. The intrinsic algebraic entropy of $\phi$ is

$$
\widetilde{\operatorname{ent}}(\phi)=\sup _{H \in \mathcal{I}_{\phi}(G)} \widetilde{\operatorname{ent}}(\phi, H) \text {. }
$$

In Section 2 we study the family $\mathcal{I}_{\phi}(G)$ of the $\phi$-inert subgroups of an Abelian group $G$, where $\phi$ is an endomorphism of $G$. In particular, we show that $\mathcal{I}_{\phi}(G)$ is a bounded sublattice of the lattice of all the subgroups of $G$ (see Lemma 2.6), containing all finite subgroups, all finite-index subgroups, as well as all $\phi$-invariant and fully invariant subgroups of $G$; but in general $\mathcal{I}_{\phi}(G)$ is wider.

In Section 3 we give the basic properties of the intrinsic algebraic entropy; some of them are similar to those of the algebraic entropy, but there are also important differences. For every endomorphism $\phi$ of any Abelian group $G$, these entropies are related by the inequalities ent $(\phi) \leq \widetilde{\operatorname{ent}}(\phi) \leq h(\phi)$; as for endomorphisms of torsion Abelian groups the equality ent $(\phi)=h(\phi)$ holds, in this case this common value coincides also with $\widetilde{\operatorname{ent}}(\phi)$. Therefore, the real novelty of intrinsic algebraic entropy is confined to the class of non-torsion Abelian groups.

The topological setting provides another motivation for introducing $\phi$-inert subgroups (see 4.1 ). Let $G$ be a locally compact Abelian group and $\mathcal{B}(G)$ be the family of all compact open subgroups of $G$. Then every $U \in \mathcal{B}(G)$ is $\phi$-inert for every continuous endomorphism $\phi: G \rightarrow G$ (i.e., $\mathcal{B}(G) \subseteq \mathcal{I}_{\phi}(G)$ ) and $\widetilde{\operatorname{ent}}(\phi, U)$ coincides with the algebraic entropy of $\phi$ with respect to $U$ (see Theorem 4.3 . In particular, $\operatorname{ent}(\phi)$ is an upper bound for the algebraic entropy of $\phi$ whenever $G$ is totally disconnected (see Remark 4.4). On the other hand, if $G$ is totally disconnected and $\phi$ is a topological automorphism of $G$, then the finite index $[U+\phi(U): U]=[\phi(U): U \cap \phi(U)]$, for $U \in \mathcal{B}(G)$, is involved also in the definition of the scale function $s(\phi)$ of Willis [26, 27] given by $s(\phi)=\min \{[\phi(U): U \cap \phi(U)]: U \in$ $\mathcal{B}(G)\}$.

In the rest of Section 4 we compute the intrinsic algebraic entropy of endomorphisms of finite dimensional $\mathbb{Q}$-vector spaces and we reveal its relation to the so-called Algebraic Yuzvinski Formula recently proved in [12. The Algebraic Yuzvinski Formula states that, for an endomorphism $\phi: \mathbb{Q}^{m} \rightarrow$ $\mathbb{Q}^{m}$ with $m \in \mathbb{N}_{+}$, the value of the algebraic entropy can be computed as

$$
h(\phi)=\log s+\sum_{\left|\lambda_{i}\right|>1} \log \left|\lambda_{i}\right|
$$

where $\left\{\lambda_{1}, \ldots, \lambda_{m}\right\} \subseteq \mathbb{C}$ are the eigenvalues of $\phi$ and $s$ is the least common multiple of the denominators of the coefficients of the characteristic polynomial of $\phi$. The term $\log s$ in this formula seems to be somewhat mysterious; now we see in Theorem 4.2 that

$$
\widetilde{\operatorname{ent}}(\phi)=\log s \text {. }
$$

In Section 5 we carefully study the particular case when the group $G$ is the $\phi$-trajectory of a finitely generated subgroup, in other words, when $G$ is a finitely generated $\mathbb{Z}[X]$-module, where the action of an integer polynomial over $G$ is induced by $\phi$. The detailed description of this case allows us to prove the Addition Theorem for the intrinsic algebraic entropy, stating that ent is an additive invariant of the category $\operatorname{Mod}(\mathbb{Z}[X])$. This result, together with the property of being upper continuous, makes the intrinsic algebraic entropy a length function on $\operatorname{Mod}(\mathbb{Z}[X])$, similarly to the algebraic entropy $h$ (see [6]).

In Section 6 we prove a Uniqueness Theorem, characterizing the intrinsic algebraic entropy in a similar fashion, mutatis mutandis, to the analogous theorems proved for the two above mentioned entropies ent and $h$ in [9] and [6], respectively. Indeed, we see that ent is the unique length function on $\operatorname{Mod}(\mathbb{Z}[X])$ coinciding with ent on torsion Abelian groups and satisfying (1.5). 
In [5] an automorphism $\phi: G \rightarrow G$ of a group $G$ is defined to be inertial if $[H: H \cap \phi(H)]$ and $[\phi(H): H \cap \phi(H)]$ are finite for every subgroup $H$ of $G$ (note that in Definition 1.1 we consider endomorphisms of Abelian groups, not only automorphisms, and we require that $H \cap \phi(H)$ has finite index only in $\phi(H)$, and not in $H$ ). Inspired by this and similarly to what is done in [4] and [18] for inner automorphisms, one can introduce a smaller class of subgroups of an Abelian group $G$, letting $\phi$ ranging in the $\operatorname{ring} \operatorname{End}(G)$ of all endomorphisms of $G$. Namely, call a subgroup $H$ of an Abelian group $G$ fully inert if $H$ is $\phi$-inert for every endomorphism $\phi$ of $G$. The family $\bigcap_{\phi \in \operatorname{End}(G)} \mathcal{I}_{\phi}(G)$ of all fully inert subgroups of $G$ contains all finite subgroups, all finite-index subgroups and all fully invariant subgroups of $G$. Fully inert subgroups are investigated in the papers [8] and [10], with particular attention paid to the Abelian groups which are fully inert in divisible groups and free groups respectively.

\section{Notation and terminology}

We denote by $\mathbb{N}, \mathbb{N}_{+}, \mathbb{P}, \mathbb{Z}$ and $\mathbb{Q}$ the set of the naturals, positive naturals, primes, integers and rationals respectively. For every $p \in \mathbb{P}$ we denote by $\mathbb{J}_{p}$ the ring of $p$-adic integers, by $\mathbb{Q}_{p}$ the field of $p$-adic numbers and by $|-|_{p}$ the $p$-adic norm on $\mathbb{Q}_{p}$; this is defined by $|p|_{p}=1 / p$ and $|q|_{p}=1$ for every $q \in \mathbb{P}$ with $q \neq p$. Let $\mathbb{R}$ be the set of real numbers, $\mathbb{R}_{\geq 0}=\{r \in \mathbb{R}: r \geq 0\}$ and $\mathbb{R}^{*}=\mathbb{R}_{\geq 0} \cup\{\infty\}$.

For an Abelian group $G$ we denote by $t(G)$ the torsion subgroup of $G$ and by $\operatorname{rk}(G)=\operatorname{dim}_{\mathbb{Q}}\left(G \otimes_{\mathbb{Z}} \mathbb{Q}\right)$ the torsion-free rank of $G$. Moreover, $D(G)$ denotes a divisible hull of $G$ and, if $G$ is torsion-free and $L$ is a subgroup of $G, L^{*}$ denotes the pure closure of $L$ in $G$. We recall that $L^{*} / L=t(G / L)$ and so $G / L^{*} \cong(G / L) /\left(L^{*} / L\right)=(G / L) / t(G / L)$, hence we have the short exact sequence

$$
0 \rightarrow L^{*} \rightarrow G \rightarrow(G / L) / t(G / L) \rightarrow 0 .
$$

For a ring $R$ we denote by $\operatorname{Mod}(R)$ the category of the left $R$-modules.

\section{Basic properties of $\phi$-inert subgroups}

In this section we prove the basic facts concerning inert subgroups, that will be used in the investigation of the intrinsic algebraic entropy. We start from the next lemma collecting some useful properties of inert subgroups.

Lemma 2.1. Let $G$ be an Abelian group, $\phi: G \rightarrow G$ an endomorphism and $H$ a $\phi$-inert subgroup of G. Then:

(a) $T_{n}(\phi, H) / H$ is finite for all $n \in \mathbb{N}_{+}$;

(b) $T(\phi, H) / H$ is torsion;

(c) if $G$ is torsion-free, then $T(\phi, H)$ is contained in $H^{*}$ and $H^{*}$ is $\phi$-invariant.

Proof. (a) We proceed by induction. For $n=2$ finiteness is ensured by definition. Let us assume that $n \geq 2$ and $T_{n}(\phi, H) / H$ is finite. The map $T_{n}(\phi, H) / H \rightarrow T_{n+1}(\phi, H) / T_{n}(\phi, H)$ induced by $\phi$ is well-defined and surjective, therefore $T_{n+1}(\phi, H) / T_{n}(\phi, H)$ is finite. Since $T_{n+1}(\phi, H) / T_{n}(\phi, H)$ is isomorphic to $\left(T_{n+1}(\phi, H) / H\right) /\left(T_{n}(\phi, H) / H\right), T_{n+1}(\phi, H) / H$ is finite as well, as an extension of $T_{n}(\phi, H) / H$ by this group.

(b) Since $T(\phi, H)=\bigcup_{n \in \mathbb{N}_{+}} T_{n}(\phi, H)$, it follows that

$$
T(\phi, H) / H=\bigcup_{n \in \mathbb{N}_{+}} T_{n}(\phi, H) / H
$$


so $T(\phi, H) / H$, as a union of finite subgroups, is torsion.

(c) The first claim is an immediate consequence of item (b), as $H^{*} / H$ is the torsion subgroup of $G / H$. To prove that $H^{*}$ is $\phi$-invariant, note that the $\phi$-trajectory $T=T(\phi, H)$ is $\phi$-invariant in $G$ and $H^{*} / T$ is torsion. If $x \in H^{*}$, then $k x \in T$ for a suitable integer $k \neq 0$. So $k \phi(x)=\phi(k x) \in T$, hence $\phi(x) \in H^{*}$, which is the pure closure of $T$ in $G$, consequently $H^{*}$ is $\phi$-invariant in $G$.

The following characterization of $\phi$-inert subgroups is needed later on.

Proposition 2.2. Let $G$ be an Abelian group, $\phi: G \rightarrow G$ an endomorphism and $H$ a subgroup of $G$. Then the following conditions are equivalent:

(a) $H$ is $\phi$-inert;

(b) there exists a finite subset $F$ of $H$ such that $T_{2}(\phi, H)=H+\phi(F)$;

(c) there exists a finite subset $F$ of $H$ such that $T_{n}(\phi, H)=H+T_{n}(\phi, F)$ for every $n \geq 2$.

If $G$ is torsion, the subset $F$ in (b) and (c) can be assumed to be a finite subgroup.

Proof. (a) $\Rightarrow(\mathrm{b})$ Since $(H+\phi(H)) / H$ is finite by hypothesis, there are finitely many cosets of $\phi(H)$ modulo $H$. Let $F$ be a finite subset of $H$, such that $H+\phi(H)=H+\phi(F)$. If $G$ is torsion and $F$ denotes the subgroup generated by these cosets, we have the same equality.

(b) $\Rightarrow(\mathrm{c})$ We induct on $n \in \mathbb{N}_{+}$. The hypothesis gives $H+\phi(H)=H+\phi(F)$ for a suitable finite subset $F$ of $H$. Assume that $T_{n}(\phi, H)=H+T_{n}(\phi, F)$ for the same finite subset $F$, and for some $n \geq 2$. Then, using that $H+\phi(H)=H+\phi(F)$ and that $H=H+F$, so also $\phi(H)=\phi(H)+\phi(F)$, we get

$$
\begin{array}{r}
T_{n+1}(\phi, H)=H+\phi\left(T_{n}(\phi, H)\right)=H+\phi(H)+\phi\left(T_{n}(\phi, F)\right)= \\
=H+\phi(F)+\phi\left(T_{n}(\phi, F)\right)=H+T_{n+1}(\phi, F) .
\end{array}
$$

We can replace "finite subset" by "finite subgroup" if $G$ is torsion.

$(\mathrm{c}) \Rightarrow(\mathrm{b}) \Rightarrow(\mathrm{a})$ are trivial.

Another useful property of inert subgroups is the following one.

Lemma 2.3. Let $G$ be an Abelian group and $\phi: G \rightarrow G$ an endomorphism. A subgroup $H$ of $G$ is $\phi$-inert if and only if $\bar{H}=(H+t(G)) / t(G)$ is $\bar{\phi}$-inert in $\bar{G}=G / t(G)$, where $\bar{\phi}: \bar{G} \rightarrow \bar{G}$ is the endomorphism induced by $\phi$, and $((H+\phi(H)) / H) \cap((H+t(G)) / H)$ is finite. The latter condition is automatically satisfied whenever $H$ is finitely generated.

Proof. Let us denote $T_{n}(\phi, H)$ by $T_{n}$ for every $n \in \mathbb{N}_{+}$. The canonical surjection $T_{n} \rightarrow\left(T_{n}+t(G)\right) / t(G)$ induces the exact sequence

$$
0 \rightarrow\left(T_{n} \cap(H+t(G))\right) / H \rightarrow T_{n} / H \rightarrow\left(T_{n}+t(G)\right) /(H+t(G)) \rightarrow 0 .
$$

By Lemma 2.1. $H$ is $\phi$-inert if and only if $T_{n} / H$ is finite; in view of the above exact sequence, this is equivalent to the facts that $\left(T_{n}+t(G)\right) /(H+t(G)) \cong\left(\left(T_{n}+t(G)\right) / t(G)\right) /((H+t(G)) / t(G))$ is finite (that is, $\bar{H}$ is $\bar{\phi}$-inert) and $\left(T_{n} \cap(H+t(G))\right) / H$ is finite. For $n=2$ this amounts to say that $((H+\phi(H)) / H) \cap((H+t(G)) / H)$ is finite; conversely, if this is true, then the same inductive argument used in Lemma 2.1 shows that $\left(T_{n} \cap(H+t(G))\right) / H$ is finite.

The last claim follows from the fact that, if $H$ is finitely generated, then $(H+\phi(H)) / H$ and its subgroups are finitely generated. So it remains to add that a finitely generated torsion Abelian group is finite. 
The next lemma will be useful in proving Theorem 4.2 .

Lemma 2.4. Let $G$ be a torsion-free Abelian group of finite rank and $\phi: G \rightarrow G$ an endomorphism. Then every finitely generated subgroup $H$ of $G$ of maximal rank is $\phi$-inert.

Proof. The quotient $(H+\phi(H)) / H$ is torsion, being a subgroup of $G / H$, and it is finitely generated being isomorphic to the quotient $\phi(H) /(H \cap \phi(H))$ of $\phi(H)$. Hence $(H+\phi(H)) / H$ is finite, as desired.

The hypothesis that $H$ is finitely generated in Lemma 2.4 cannot be removed, as shown in 8 Example 2.5]. Obviously, Lemma 2.4 fails also if we do not assume that the finitely generated subgroup $H$ of $G$ has maximal rank; in fact, if $\operatorname{rk}(H)<\operatorname{rk}(G)$, it can happen that $\phi(H)$ is not contained in the pure closure $H^{*}$ of $H$, and this implies that $H$ is not $\phi$-inert.

The next proposition partially reverses item (c) of Lemma 2.1 for finitely generated subgroups.

Proposition 2.5. Let $G$ be a torsion-free Abelian group, $\phi: G \rightarrow G$ an endomorphism and $H$ a finitely generated subgroup of $G$. Then $H$ is $\phi$-inert if and only if $H^{*}$ is $\phi$-invariant.

Proof. By Lemma 2.1(c), we need only to prove that if $H^{*}$ is $\phi$-invariant then $H$ is $\phi$-inert. As $H$ and $H^{*}$ have the same rank, Lemma 2.4 implies that $H$ is $\phi$-inert in $H^{*}$, hence it is also $\phi$-inert in $G$.

The following lemma shows that $\mathcal{I}_{\phi}(G)$ is a sublattice of the lattice of all the subgroups of $G$ (this lattice is bounded, as 0 and $G$ are always $\phi$-inert).

Lemma 2.6. Let $G$ be an Abelian group, $\phi: G \rightarrow G$ an endomorphism, $H$ and $H^{\prime}$ two $\phi$-inert subgroups of $G$. Then $H \cap H^{\prime}$ and $H+H^{\prime}$ are both $\phi$-inert.

Proof. The embeddings

$$
\begin{aligned}
\frac{\left(H \cap H^{\prime}\right)+\phi\left(H \cap H^{\prime}\right)}{H \cap H^{\prime}} & \rightarrow \frac{\left(H \cap H^{\prime}\right)+\phi\left(H \cap H^{\prime}\right)+H}{H} \oplus \frac{\left(H \cap H^{\prime}\right)+\phi\left(H \cap H^{\prime}\right)+H^{\prime}}{H^{\prime}} \\
& \subseteq \frac{H+\phi(H)}{H} \oplus \frac{H^{\prime}+\phi\left(H^{\prime}\right)}{H^{\prime}} .
\end{aligned}
$$

imply that $H \cap H^{\prime}$ is $\phi$-inert. Concerning $H+H^{\prime}$, we note that

$$
\frac{H+H^{\prime}+\phi\left(H+H^{\prime}\right)}{H+H^{\prime}}=\frac{H+\phi(H)+H^{\prime}}{H+H^{\prime}}+\frac{H^{\prime}+\phi\left(H^{\prime}\right)+H}{H+H^{\prime}}
$$

is a quotient of the finite group $((H+\phi(H)) / H) \oplus\left(\left(H^{\prime}+\phi\left(H^{\prime}\right)\right) / H^{\prime}\right)$, hence also $H+H^{\prime}$ is $\phi$-inert.

A last useful property of $\phi$-inert subgroups is contained in the next lemma.

Lemma 2.7. Let $G$ be an Abelian group and $\phi: G \rightarrow G$ an endomorphism. If $H$ is $\phi$-inert, then for every $n \in \mathbb{N}_{+}$:

(a) $\phi^{n}(H)$ is $\phi$-inert;

(b) $H$ is $\phi^{n}$-inert.

Proof. Let $n \in \mathbb{N}_{+}$. To prove (a) it suffices to note that the homomorphism $H+\phi(H) / H \rightarrow \phi^{n}(H)+$ $\phi^{n+1}(H) / \phi^{n}(H)$ induced by $\phi^{n}$ is surjective. To verify (b) observe that $H+\phi^{n}(H) \subseteq T_{n}(\phi, H)$; since $T_{n}(\phi, H) / H$ is finite by Lemma 2.1, also $H+\phi^{n}(H) / H$ is finite.

Lemma 2.7(b) shows that for an endomorphism $\phi: G \rightarrow G$ of an Abelian group $G$, a subgroup $H$ of $G$ is $\phi$-inert if and only if $H$ is $\phi^{n}$-inert for every $n \in \mathbb{N}_{+}$. In other words,

$$
\mathcal{I}_{\phi}(G)=\bigcap_{n \in \mathbb{N}_{+}} \mathcal{I}_{\phi^{n}}(G) .
$$

If $\phi: G \rightarrow G$ is an automorphism, then $\mathcal{I}_{\phi}(G) \neq \mathcal{I}_{\phi^{-1}}(G)$ may occur (see Example 3.1(b)). 


\section{The intrinsic algebraic entropy and its properties}

We start this section introducing the following basic example concerning the Bernoulli shifts.

Example 3.1. For an arbitrary Abelian group define the right Bernoulli shift $\beta_{G}: G^{(\mathbb{N})} \rightarrow G^{(\mathbb{N})}$ by $\beta_{G}\left(x_{0}, x_{1}, \ldots\right)=\left(0, x_{0}, x_{1}, \ldots\right)$, and the two-sided (right) Bernoulli shift $\sigma_{G}: G^{(\mathbb{Z})} \rightarrow G^{(\mathbb{Z})}$ by $\sigma_{G}\left(\left(x_{n}\right)_{n \in \mathbb{Z}}\right)=\left(x_{n-1}\right)_{n \in \mathbb{Z}}$. Note that $\sigma_{G}$ is an automorphism, while $\beta_{G}$ is a non-surjective monomorphism.

(a) For $p$ a prime, for the two-sided Bernoulli shift $\sigma_{\mathbb{Z}(p)}: \mathbb{Z}(p)^{(\mathbb{Z})} \rightarrow \mathbb{Z}(p)^{(\mathbb{Z})}$ the subgroup $H=$ $\bigoplus_{-\infty}^{0} \mathbb{Z}(p)$ is infinite and $\sigma_{\mathbb{Z}(p)}$-inert.

(b) For the two-sided Bernoulli shift $\sigma_{\mathbb{Z}}: \mathbb{Z}^{(\mathbb{Z})} \rightarrow \mathbb{Z}^{(\mathbb{Z})}$ the subgroup $H=\bigoplus_{0}^{\infty} \mathbb{Z}$ if $\sigma_{\mathbb{Z}}$-invariant, hence $\sigma_{\mathbb{Z}}$-inert, but it is not $\sigma_{\mathbb{Z}}^{-1}$-inert.

\subsection{Examples and comparison with the algebraic entropy}

The following lemma shows that the limit defining the intrinsic algebraic entropy exists and it is finite.

Lemma 3.2. Let $G$ be an Abelian group, $\phi: G \rightarrow G$ an endomorphism and $H$ a $\phi$-inert subgroup of G. Then the limit

$$
\lim _{n \rightarrow \infty} \frac{\log \left|T_{n}(\phi, H) / H\right|}{n}
$$

exists and it is finite.

Proof. In order to simplify notation, we set $T_{n}=T_{n}(\phi, H)$ for each $n \in \mathbb{N}_{+}$. Being $H$ a $\phi$-inert subgroup of $G, T_{n} / H$ is finite for each $n \in \mathbb{N}_{+}$by Lemma 2.1(a). Set

$$
\alpha_{n+1}=\frac{\left|T_{n+1} / H\right|}{\left|T_{n} / H\right|}=\left|\frac{T_{n+1}}{T_{n}}\right|=\left|\frac{\phi^{n}(H)}{T_{n} \cap \phi^{n}(H)}\right| .
$$

For each $n \geq 2$, the group $\phi^{n}(H) /\left(T_{n} \cap \phi^{n}(H)\right)$ is a quotient of the group

$$
B_{n}=\phi^{n}(H) /\left(\phi\left(T_{n-1}\right) \cap \phi^{n}(H)\right) \cong \phi\left(T_{n}\right) / \phi\left(T_{n-1}\right) \cong T_{n} /\left(T_{n-1}+\left(T_{n} \cap \operatorname{ker} \phi\right)\right) .
$$

Therefore $\alpha_{n+1}$ divides $\beta_{n}=\left|B_{n}\right|$. On the other hand, since the latter group is a quotient of $\frac{T_{n} / H}{T_{n-1} / H}$, we get that $\beta_{n}$ divides $\alpha_{n}$ so, in particular, $\alpha_{n+1}$ divides $\alpha_{n}$. We conclude that the sequence of nonnegatve integers $\left(\alpha_{n}\right)_{n \in \mathbb{N}_{+}}$is non-increasing, hence it is stationary. So, as in case of $H$ finite (see [9, Proposition 1.3]), it follows that

$$
\lim _{n \rightarrow \infty} \frac{\log \left|T_{n}(\phi, H) / H\right|}{n}=\log \alpha,
$$

where $\alpha=\alpha_{n}$ for all $n$ large enough.

Now we give a characterization of the $\phi$-inert subgroups $H$ with $\widetilde{\operatorname{ent}}(\phi, H)=0$ :

Proposition 3.3. Let $\phi: G \rightarrow G$ be an endomorphism of an Abelian group $G$ and $H$ a $\phi$-inert subgroup of $G$. The following conditions are equivalent:

(a) $\widetilde{\operatorname{ent}}(\phi, H)=0$;

(b) $H$ has finite index in its $\phi$-trajectory $T(\phi, H)$. 
Consequently $\widetilde{\operatorname{ent}}(\phi)=0$ if and only if every $\phi$-inert subgroup of $G$ has finite index in its $\phi$-trajectory. In particular, $\widetilde{\operatorname{ent}}(\phi)=0$ if all subgroups of $G$ are $\phi$-invariant.

Proof. The proof of Lemma 3.2 shows that $\widetilde{\operatorname{ent}}(\phi, H)=\log \alpha_{n}$ for all $n \in \mathbb{N}_{+}$large enough, where $\alpha_{n}=$ $\left|T_{n+1}(\phi, H) / T_{n}(\phi, H)\right|$. As $T(\phi, H)=\bigcup_{n \in \mathbb{N}_{+}} T_{n}(\phi, H)$, ent $(\phi, H)=0$ exactly if $T(\phi, H)=T_{n}(\phi, H)$ for some $n \in \mathbb{N}_{+}$. Since $H$ has finite index in $T_{n}(\phi, H)$ by Lemma 2.1(a), the equivalence of (a) and (b) follows. The last claim is an immediate consequence.

Proposition 3.3 shows in particular that $\widetilde{\operatorname{ent}}(\phi, H)=0$ for every subgroup $H$ of finite index in $G$.

We examine the basic examples of the endomorphisms of rational groups. Here and in the sequel we make use of the well known fact that, given a torsion-free Abelian group $H$ of finite rank $n$, for each $b \in \mathbb{N}_{+}$the cardinality of $H / b H$ is at most $b^{n}$ ([1, Theorem 0.1]); in particular, $H / b H$ is finite.

Example 3.4. (1) Let $\phi: \mathbb{Z} \rightarrow \mathbb{Z}$ be a non-zero endomorphism of the group of the integers. Trivially, $\mathbb{Z}$ has no non-trivial finite subgroups, hence ent $(\phi)=0$. As $\phi$ acts as the multiplication by a non-zero integer, every subgroup of $\mathbb{Z}$ is $\phi$-invariant, so Proposition 3.3 implies that $\widetilde{\operatorname{ent}}(\phi)=0$.

(2) Let $\phi: \mathbb{Q} \rightarrow \mathbb{Q}$ be a non-zero endomorphism of the group of the rational numbers, and note that $\mathbb{Q}$ has no non-trivial finite subgroups, hence, as before, ent $(\phi)=0$. As $\phi$ acts as the multiplication by a non-zero fraction $a / b$, with $b>0$ and $a$ and $b$ relatively prime, a non-zero subgroup $H$ of $\mathbb{Q}$ is $\phi$-invariant exactly if $a H \leq b H$. This inequality is equivalent to the equality $H=b H$; in fact, $H=b H$ trivially implies $a H \leq b H$, and to see that the converse holds recall that $(a, b)=1$ implies $a H+b H=H$. Hence $H$ is $\phi$-invariant exactly if it is $p$-divisible by all primes $p$ dividing $b$. Furthermore, if $H$ is an arbitrary subgroup of $\mathbb{Q}$, we have:

$$
\frac{H+\phi(H)}{H}=\frac{H+a b^{-1} H}{H} \cong \frac{b H+a H}{b H}=\frac{H}{b H} ;
$$

hence $H$ is $\phi$-inert, since $H / b H$ is finite. Setting $m=|H / b H|$, by the fact quoted above we know that $m \leq b$; it easy to show that $\widetilde{\operatorname{ent}}(\phi, H)=\log m \leq \log b$ and consequently $\widetilde{\operatorname{ent}}(\phi)=$ $\widetilde{\operatorname{ent}}(\phi, \mathbb{Z})=\log b$. This example is extended to higher dimensions in the next Theorem 4.2 . Recall that $h(\phi)=\max \{\log |a|, \log b\}$ (see [6] for a direct computation, otherwise one can apply the Algebraic Yuzvinski Formula proved in [12]). Hence, $h(\phi)>\widetilde{\operatorname{ent}}(\phi)$ in case $|a|>b$.

(3) Generalizing the two preceding cases, let $\phi: G \rightarrow G$ be a non-zero endomorphism of a fixed subgroup $G$ of the rational numbers. We can assume, without loss of generality, that $\mathbb{Z} \subseteq G$. As $\phi$ acts as the multiplication by a non-zero fraction $a / b$ (with $(a, b)=1$ ) belonging to the maximal subring contained in $G$, the same argument used in case (2) shows that $\widetilde{\operatorname{ent}}(\phi)=\log b$.

An easy property of the intrinsic algebraic entropy is that $\widetilde{\operatorname{ent}}(\phi)=\widetilde{\operatorname{ent}}(-\phi)$. On the other hand, $\widetilde{\text { ent }}$ is not preserved by taking the inverse of a given automorphism, as underlined in the following remark.

Remark 3.5. In case (2) of the preceding example, the automorphism $\phi^{-1}$ of $\mathbb{Q}$ acts as the multiplication by $b / a$, hence ent $\left(\phi^{-1}\right)=\log |a|$. This shows that the equalities ent $(\phi)=\operatorname{ent}\left(\phi^{-1}\right)$ and $h(\phi)=h\left(\phi^{-1}\right)$, which hold for an automorphism $\phi$, do not extend from the algebraic entropies to the intrinsic algebraic entropy.

We would like to compare the intrinsic algebraic entropy ent with the algebraic entropy ent and with the algebraic entropy $h$. In contrast with the inequality $h(\phi)>\widetilde{\operatorname{ent}}(\phi)$ established in Example $3.4(2)$, we see now that always $h(\phi)=\widetilde{\operatorname{ent}}(\phi)$ in case the Abelian group $G$ is torsion. 
Proposition 3.6. Let $G$ be an Abelian group and $\phi: G \rightarrow G$ an endomorphism. Then ent $(\phi) \leq$ $\widetilde{\operatorname{ent}}(\phi) \leq h(\phi)$. If $G$ is torsion, then $\operatorname{ent}(\phi)=\widetilde{\operatorname{ent}}(\phi)=h(\phi)$.

Proof. Since the family $\mathcal{I}_{\phi}(G)$ of the $\phi$-inert subgroups of $G$ contains the family $\mathcal{F}(G)$ of the finite subgroups of $G$, one obviously derives the inequality $\operatorname{ent}(\phi) \leq \widetilde{\operatorname{ent}}(\phi)$. Let $H$ be a $\phi$-inert subgroup. According to Proposition 2.2, there exists a finite subset $F$ of $H$ such that $T_{n}(\phi, H)=H+T_{n}(\phi, F)$ for all $n \in \mathbb{N}_{+}$. Then $\left|T_{n}(\phi, H) / H\right|=\left|\left(H+T_{n}(\phi, F)\right) / H\right|$. Taking logarithms and dividing by $n$, the limit on the left hand side gives ent $(\phi, H)$. On the other hand, $\left|H+T_{n}(\phi, F) / H\right| \leq\left|T_{n}(\phi, F)\right|$, thus $\log \mid H+$ $T_{n}(\phi, F) / H|\leqq \log | T_{n}(\phi, F) \mid$ and after dividing by $n$ we get ent $(\phi, H) \leq \lim _{n \rightarrow \infty} \log \left|T_{n}(\phi, F)\right| / n \leq$ $h(\phi)$, hence $\operatorname{ent}(\phi) \leq h(\phi)$. If $G$ is torsion, it is well known (see [6] or [20]) that ent $(\phi)=h(\phi)$, hence the latter claim follows.

In Theorem 4.3 and Remark 4.4 we find a counterpart of the equality $\operatorname{ent}(\phi)=\widetilde{\operatorname{ent}}(\phi)$ in the case of a continuous endomorphism $\phi$ of a locally compact totally disconnected Abelian group $G$ covered by compact subgroups (note that if such a group $G$ is discrete, then it is torsion, so we obtain the case considered above).

Proposition 3.6 shows that for torsion Abelian groups it suffices to compute the intrinsic algebraic entropy with respect to finite subgroups, even if they may be less than the inert ones, as Example 3.1 shows.

As the Bernoulli shifts are crucial examples of endomorphisms in the theory of algebraic entropy, it is convenient to examine what happens also for their intrinsic algebraic entropy.

Example 3.7. We prove that $\widetilde{\operatorname{ent}}\left(\beta_{G}\right)=\log |G|$ for the right Bernoulli shift $\beta_{G}: G^{(\mathbb{N})} \rightarrow G^{(\mathbb{N})}$ (see Example 3.1), where we intend that $\log |G|=\infty$ in case $G$ is infinite. If $G$ is torsion, this follows from Proposition 3.6 and the equality $\operatorname{ent}\left(\beta_{G}\right)=\log |G|$ proved in [9, Example 1.9].

Now assume that $G$ is a non-torsion Abelian group and choose a non-torsion element $g \in G$. Consider the element $x=(g, 0,0, \ldots) \in G^{(\mathbb{N})}$ and fix an integer $k>1$. Let the subgroup $H_{k}$ of $G^{(\mathbb{N})}$ be defined as follows:

$$
H_{k}=x \mathbb{Z}+T\left(\beta_{G}, k x \mathbb{Z}\right)=g \mathbb{Z} \oplus \bigoplus_{i \geq 1} k \beta_{G}^{i}(x) \mathbb{Z} .
$$

Then, for every $n \in \mathbb{N}_{+}$, we get:

$$
T_{n}\left(\beta_{G}, H_{k}\right)=\bigoplus_{i<n} \beta_{G}^{i}(x) \mathbb{Z} \oplus \bigoplus_{i \geq n} k \beta_{G}^{i}(x) \mathbb{Z} .
$$

It follows that $T_{n}\left(\beta_{G}, H_{k}\right) / H_{k}$ is isomorphic to the direct sum of $n-1$ many copies of $\mathbb{Z} / k \mathbb{Z}$, so it has size $k^{n-1}$. Thus, every subgroup $H_{k}$ is $\beta_{G}$-inert, $\widetilde{\operatorname{ent}}\left(\beta_{G}, H_{k}\right)=\log k$, and consequently $\widetilde{\operatorname{ent}}\left(\beta_{G}\right)=\infty$.

As in the case of the algebraic entropy ent, the range of the intrinsic algebraic entropy is a discrete subset of the real numbers; in fact, if it is finite, it is reached by a suitable inert subgroup as a maximum:

Lemma 3.8. Let $G$ be an Abelian group and $\phi: G \rightarrow G$ an endomorphism. If $\widetilde{\operatorname{ent}}(\phi)<\infty$, then there exists a $\phi$-inert subgroup $H$ of $G$ such that $\widetilde{\operatorname{ent}}(\phi)=\widetilde{\operatorname{ent}}(\phi, H)=\log n$ for some $n \in \mathbb{N}_{+}$.

Proof. By the proof of Lemma 3.2, for every $H \in \mathcal{I}_{\phi}(G)$ there exists a positive integer $n_{H}$ such that $\widetilde{\operatorname{ent}}(\phi, H)=\log n_{H}$. Consider the set $\mathcal{F}=\left\{\widetilde{\operatorname{ent}}(\phi, H): H \in \mathcal{I}_{\phi}(G)\right\} \subseteq\left\{\log n: n \in \mathbb{N}_{+}\right\}$, and notice that $\mathcal{F}$ is bounded by the finite value $\widetilde{\operatorname{ent}}(\phi)$. One can easily conclude that $\mathcal{F}$ is finite and then $\widetilde{\operatorname{ent}}(\phi)=\sup _{H \in \mathcal{I}_{\phi}(G)} \log n_{H}=\max \mathcal{F}$ is an element of $\mathcal{F}$. 
A consequence of the above lemma is that the set of finite values of ent ( considered as a function from the class of all the endomorphisms of abelian groups) is contained in the subset $\left\{\log n: n \in \mathbb{N}_{+}\right\}$of $\mathbb{R}_{\geq 0}$. Moreover, these two sets actually coincide, since by Example 3.7 we know that $\widetilde{\operatorname{ent}}\left(\beta_{G}\right)=\log |G|$ if $G$ is a finite Abelian group.

\subsection{Typical properties of entropy functions}

In the rest of this section we provide some fundamental properties of the intrinsic algebraic entropy that will allow us to prove the Uniqueness Theorem in Section 6. Similar results hold for the algebraic entropies ent and $h$ (see [25], [9] and [6]); however, in the present situation, ad hoc arguments are needed.

In the next lemma we establish invariance of the intrinsic algebraic entropy under conjugation.

Lemma 3.9. Let $\phi: G \rightarrow G$ be an endomorphism and $\xi: G \rightarrow G^{\prime}$ an isomorphism of Abelian groups. Then $\widetilde{\operatorname{ent}}(\phi)=\widetilde{\operatorname{ent}}\left(\xi \phi \xi^{-1}\right)$.

Proof. Set $\psi=\xi \phi \xi^{-1}$. A subgroup $H$ of $G$ is $\phi$-inert if and only if $\xi(H)$ is $\psi$-inert in $G^{\prime}$, and an easy computation shows that $\left|T_{n}(\phi, H) / H\right|=\left|T_{n}(\psi, \xi(H)) / \xi(H)\right|$ for every $n \in \mathbb{N}_{+}$. Therefore $\widetilde{\operatorname{ent}}(\xi, H)=\widetilde{\operatorname{ent}}(\psi, \xi(H))$ and the desired equality follows.

The next lemma establishes monotonicity of the intrinsic algebraic entropy under taking restrictions and quotients with respect to a $\phi$-invariant subgroup.

Lemma 3.10. Let $G$ be an Abelian group, $\phi: G \rightarrow G$ an endomorphism and $H$ a $\phi$-invariant subgroup of $G$. Then:

(a) $\widetilde{\operatorname{ent}}(\phi) \geq \widetilde{\operatorname{ent}}\left(\phi \uparrow_{H}\right)$ and $\widetilde{\operatorname{ent}}(\phi) \geq \widetilde{\operatorname{ent}}(\bar{\phi})$, where $\bar{\phi}: G / H \rightarrow G / H$ is the endomorphism induced by $\phi$;

(b) if $K$ is a $\phi$-inert subgroup of $G$ and $\bar{K}=(K+H) / H$, then $K \cap H$ is $\phi \uparrow_{H \text {-inert }}, \bar{K}$ is $\bar{\phi}$-inert and

$$
\widetilde{\operatorname{ent}}(\phi, K) \geq \widetilde{\operatorname{ent}}\left(\phi \uparrow_{H}, H \cap K\right)+\widetilde{\operatorname{ent}}(\bar{\phi}, \bar{K}) .
$$

Proof. (a) The first inequality is obvious, since a $\phi \uparrow_{H}$-inert subgroup of $H$ is also a $\phi$-inert subgroup of $G$. Let now $\bar{K}=K / H$ be a $\bar{\phi}$-inert subgroup of $G / H$, with $H \leq K \leq G$. Since

$$
\frac{T_{n}(\bar{\phi}, \bar{K})}{\bar{K}} \cong \frac{T_{n}(\phi, K)}{K}
$$

for all $n \in \mathbb{N}_{+}, K$ is $\phi$-inert in $G$ and $\widetilde{\operatorname{ent}}(\bar{\phi}, \bar{K})=\widetilde{\operatorname{ent}}(\phi, K) \leq \widetilde{\operatorname{ent}}(\phi)$. Hence the latter inequality also holds.

(b) It is easy to check that the following short sequence is exact for all $n \geq 1$ :

$$
0 \rightarrow \frac{T_{n}(\phi, K) \cap(K+H)}{K} \rightarrow \frac{T_{n}(\phi, K)}{K} \rightarrow \frac{T_{n}(\phi, K)+H}{K+H} \rightarrow 0 .
$$

The last non-zero term in the above sequence is isomorphic to $T_{n}(\bar{\phi}, \bar{K}) / \bar{K}$, because $\left(T_{n}(\phi, K)+\right.$ $H) / H=T_{n}(\bar{\phi}, \bar{K})$. The first non-zero term contains the subgroup

$$
\frac{\left(K+T_{n}\left(\phi \uparrow_{H}, H \cap K\right)\right) \cap(K+H)}{K} \cong \frac{T_{n}\left(\phi \uparrow_{H}, H \cap K\right)}{(H \cap K)} .
$$

Consequently we deduce that

$$
\left|T_{n}(\phi, K) / K\right| \geq\left|T_{n}(\bar{\phi}, \bar{K}) / \bar{K}\right| \cdot\left|T_{n}\left(\phi \uparrow_{H}, H \cap K\right) /(H \cap K)\right| \cdot
$$

Taking logarithms, dividing by $n$ and making $n$ tend to $\infty$, we get the desired inequality. 
We apply the next lemma in the proof of Lemma 3.12 below.

Lemma 3.11. Let $G$ be an Abelian group, $\phi: G \rightarrow G$ an endomorphism and $H$ a subgroup of $G$.

(a) If $H$ is $\phi$-inert and $H^{\prime}=T_{k}(\phi, H)$ for some $k \in \mathbb{N}_{+}$, then $H^{\prime}$ is $\phi$-inert and $\widetilde{\operatorname{ent}}(\phi, H)=$ $\operatorname{ent}\left(\phi, H^{\prime}\right)$.

(b) If $H$ is $\phi^{k}$-inert for some $k \in \mathbb{N}_{+}$and $H^{\prime}=T_{k}(\phi, H)$, then $H^{\prime}$ is $\phi$-inert (so, in particular $\phi^{k}$-inert) and $\widetilde{\operatorname{ent}}\left(\phi^{k}, H\right)=\widetilde{\operatorname{ent}}\left(\phi^{k}, H^{\prime}\right)$.

Proof. (a) Since $H^{\prime}+\phi\left(H^{\prime}\right)=T_{k+1}(\phi, H)$, we have $\left(H^{\prime}+\phi\left(H^{\prime}\right)\right) / H^{\prime}=T_{k+1}(\phi, H) / T_{k}(\phi, H)$. This quotient is finite, as it is a quotient of $T_{k+1}(\phi, H) / H$, which is finite by the assumption that $H$ is $\phi$-inert and Lemma 2.1(a). Moreover, since

$$
\left|\frac{T_{n}\left(\phi, H^{\prime}\right)}{H^{\prime}}\right|=\left|\frac{T_{k+n}(\phi, H)}{H^{\prime}}\right|=\left|\frac{T_{k+n}(\phi, H)}{H}\right| \cdot\left|H^{\prime} / H\right|,
$$

and $\left|H^{\prime} / H\right|$ is a finite constant by Lemma 2.1(a), it follows that

$$
\widetilde{\operatorname{ent}}\left(\phi, H^{\prime}\right)=\lim _{n \rightarrow \infty} \frac{\log \left|T_{n}\left(\phi, H^{\prime}\right) / H^{\prime}\right|}{n}=\lim _{n \rightarrow \infty} \frac{\log \left|T_{k+n}(\phi, H) / H\right|}{n}=\widetilde{\operatorname{ent}}(\phi, H) .
$$

(b) To prove that $H^{\prime}$ is $\phi$-inert, we need to verify that $\left(H^{\prime}+\phi\left(H^{\prime}\right)\right) / H^{\prime}$ is finite. Since $H^{\prime}+\phi\left(H^{\prime}\right)=$ $T_{k+1}(\phi, H)=H^{\prime}+\phi^{k}(H)$, we have that $\left(H^{\prime}+\phi\left(H^{\prime}\right)\right) / H^{\prime} \cong \phi^{k}(H) /\left(H^{\prime} \cap \phi^{k}(H)\right)$. This is finite since it is a quotient of $\phi^{k}(H) /\left(H \cap \phi^{k}(H)\right) \cong\left(H+\phi^{k}(H)\right) / H$, which is finite by the assumption that $H$ is $\phi^{k}$-inert. Hence, $H^{\prime}$ is $\phi$-inert. By Lemma 2.7 the subgroup $H^{\prime}$ is also $\phi^{k}$-inert. Item (a) gives that $\widetilde{\operatorname{ent}}\left(\phi^{k}, H\right)=\widetilde{\operatorname{ent}}\left(\phi^{k}, H^{\prime}\right)$.

The next lemma establishes a "logarithmic law" for the intrinsic algebraic entropy.

Lemma 3.12. Let $G$ be an Abelian group and $\phi: G \rightarrow G$ an endomorphism. Then $\widetilde{\operatorname{ent}}\left(\phi^{k}\right)=k \cdot \widetilde{\operatorname{ent}}(\phi)$ for every $k \in \mathbb{N}_{+}$.

Proof. Fix $k \in \mathbb{N}_{+}$. First we prove the inequality $\widetilde{\operatorname{ent}}\left(\phi^{k}\right) \leq k \cdot \widetilde{\operatorname{ent}}(\phi)$. Let $H$ be a $\phi^{k}$-inert subgroup of $G$ and $H^{\prime}=T_{k}(\phi, H)$. Lemma 3.11 (b) yields that $H^{\prime}$ is $\phi$-inert, so also $\phi^{k}$-inert, and $\widetilde{\operatorname{ent}}\left(\phi^{k}, H\right)=$ $\widetilde{\operatorname{ent}}\left(\phi^{k}, H^{\prime}\right)$.

Let $n \in \mathbb{N}_{+}$. Then $T_{n}\left(\phi^{k}, H^{\prime}\right) \subseteq T_{k n-k+1}\left(\phi, H^{\prime}\right)$ and so

$$
\begin{aligned}
\widetilde{\operatorname{ent}}\left(\phi^{k}, H^{\prime}\right) & =\lim _{n \rightarrow \infty} \frac{\log \left|T_{n}\left(\phi^{k}, H^{\prime}\right) / H^{\prime}\right|}{n} \leq \lim _{n \rightarrow \infty} \frac{\log \left|T_{k n-k+1}\left(\phi, H^{\prime}\right) / H^{\prime}\right|}{n} \\
& =\lim _{n \rightarrow \infty} \frac{\log \left|T_{k n-k+1}\left(\phi, H^{\prime}\right) / H^{\prime}\right|}{k n-k+1} \cdot \lim _{n \rightarrow \infty} \frac{k n-k+1}{n} \\
& =k \cdot \lim _{n \rightarrow \infty} \frac{\log \left|T_{k n-n+1}\left(\phi, H^{\prime}\right) / H^{\prime}\right|}{k n-k+1}=k \cdot \widetilde{\operatorname{ent}}\left(\phi, H^{\prime}\right) .
\end{aligned}
$$

Therefore, $\widetilde{\operatorname{ent}}\left(\phi^{k}, H\right)=\widetilde{\operatorname{ent}}\left(\phi^{k}, H^{\prime}\right) \leq k \cdot \widetilde{\operatorname{ent}}\left(\phi, H^{\prime}\right)$. Since $H^{\prime}$ is $\phi$-inert, we can conclude that $\widetilde{\operatorname{ent}}\left(\phi^{k}\right) \leq k \cdot \widetilde{\operatorname{ent}}(\phi)$.

To prove the converse inequality, let $H$ be a $\phi$-inert subgroup of $G$ and $H_{1}=T_{k}(\phi, H)$. Moreover, let $n \in \mathbb{N}_{+}$. By Lemma 3.11(a) the subgroup $H_{1}$ is $\phi$-inert and $H_{1} / H$ is finite. By Lemma 2.7 the subgroup $H_{1}$ is also $\phi^{k}$-inert. Moreover, $T_{n}\left(\phi^{k}, H_{1}\right)=T_{k n}(\phi, H)$ and so $\left|T_{n}\left(\phi^{k}, H_{1}\right) / H_{1}\right| \cdot\left|H_{1} / H\right|=$ $\left|T_{k n}(\phi, H) / H\right|$. Then, as $\left|H_{1} / H\right|$ is constant,

$$
\frac{1}{k} \cdot \widetilde{\operatorname{ent}}\left(\phi^{k}, H_{1}\right)=\lim _{n \rightarrow \infty} \frac{\log \left|T_{n}\left(\phi^{k}, H_{1}\right) / H_{1}\right|}{k n}=\lim _{n \rightarrow \infty} \frac{\log \left|T_{k n}(\phi, H) / H\right|}{k n}=\widetilde{\operatorname{ent}}(\phi, H) .
$$

We can conclude that $\widetilde{\operatorname{ent}}\left(\phi^{k}\right) \geq k \cdot \widetilde{\operatorname{ent}}(\phi)$. 
Here we obtain a weaker form of the Addition Theorem with respect to direct sums (the general case is discussed in Section 5).

Lemma 3.13. Let $G_{i}$ be Abelian groups and $\phi_{i}: G_{i} \rightarrow G_{i}$ endomorphisms for $i=1,2$. Then $\widetilde{\operatorname{ent}}\left(\phi_{1} \oplus \phi_{2}\right)=\widetilde{\operatorname{ent}}\left(\phi_{1}\right)+\widetilde{\operatorname{ent}}\left(\phi_{2}\right)$.

Proof. Let $H_{i}$ be a $\phi_{i}$-inert subgroup of $G_{i}$, for $i=1,2$. Setting $\phi=\phi_{1} \oplus \phi_{2}$, for every $n \in \mathbb{N}_{+}$we have $T_{n}\left(\phi, H_{1} \oplus H_{2}\right) /\left(H_{1} \oplus H_{2}\right) \cong\left(T_{n}\left(\phi_{1}, H_{1}\right) / H_{1}\right) \oplus\left(T_{n}\left(\phi_{2}, H_{2}\right) / H_{2}\right)$, therefore $H_{1} \oplus H_{2}$ is $\phi$-inert and

$$
\widetilde{\operatorname{ent}}\left(\phi, H_{1} \oplus H_{2}\right)=\widetilde{\operatorname{ent}}\left(\phi_{1}, H_{1}\right)+\widetilde{\operatorname{ent}}\left(\phi_{2}, H_{2}\right) .
$$

Let $H$ be a $\phi$-inert subgroup of $G=G_{1} \oplus G_{2}, p_{i}: G \rightarrow G_{i}$ the canonical projection and $H_{i}=p_{i}(H)$, for $i=1,2$. Each $H_{i}$ is a $\phi_{i}$-inert subgroup of $G_{i}$, as the homomorphism $(H+\phi(H)) / H \rightarrow\left(H_{i}+\phi_{i}\left(H_{i}\right)\right) / H_{i}$ induced by $p_{i}$ is well-defined and surjective. Therefore $H_{1} \oplus H_{2}$ is a $\phi$-inert subgroup of $G$. Since for every $n \in \mathbb{N}_{+}$the homomorphism $T_{n}(\phi, H) / H \rightarrow T_{n}\left(\phi, H_{1} \oplus H_{2}\right) /\left(H_{1} \oplus H_{2}\right)$ induced by $p_{1} \oplus p_{2}$ is welldefined and injective, $\widetilde{\operatorname{ent}}(\phi, H) \leq \widetilde{\operatorname{ent}}\left(\phi, H_{1} \oplus H_{2}\right)$. By (3.1) we can conclude that $\widetilde{\operatorname{ent}}(\phi) \leq \widetilde{\operatorname{ent}}\left(\phi_{1}\right)+$ $\widetilde{\operatorname{ent}}\left(\phi_{2}\right)$. If $\widetilde{\operatorname{ent}}(\phi)=\infty$, by the latter inequality we can conclude that $\widetilde{\operatorname{ent}}(\phi)=\widetilde{\operatorname{ent}}\left(\phi_{1}\right)+\widetilde{\operatorname{ent}}\left(\phi_{2}\right)$. So assume that $\widetilde{\operatorname{ent}}(\phi)$ is finite. By Lemma $3.10($ a $)$ we have that $\widetilde{\operatorname{ent}}\left(\phi_{1}\right)$ and $\widetilde{\operatorname{ent}}\left(\phi_{2}\right)$ are finite as well. For $i=1,2$, let $H_{i}$ be a $\phi_{i}$-inert subgroup of $G_{i}$ such that $\widetilde{\operatorname{ent}}\left(\phi_{i}\right)=\widetilde{\operatorname{ent}}\left(\phi_{i}, H_{i}\right)$. Then (3.1) gives

$$
\widetilde{\operatorname{ent}}(\phi) \geq \widetilde{\operatorname{ent}}\left(\phi, H_{1} \oplus H_{2}\right)=\widetilde{\operatorname{ent}}\left(\phi_{1}, H_{1}\right)+\widetilde{\operatorname{ent}}\left(\phi_{2}, H_{2}\right)=\widetilde{\operatorname{ent}}\left(\phi_{1}\right)+\widetilde{\operatorname{ent}}\left(\phi_{2}\right),
$$

and this concludes the proof.

The next lemma shows that the intrinsic algebraic entropy is "continuous" with respect to direct limits.

Lemma 3.14. Let $G$ be an Abelian group and $\phi: G \rightarrow G$ an endomorphism, and assume that the group $G$ is the direct limit of a family of $\phi$-invariant subgroups $\left\{G_{i}: i \in I\right\}$. Let $\phi_{i}=\phi\left\lceil G_{i}\right.$ for all $i \in I$. Then $\widetilde{\operatorname{ent}}(\phi)=\sup _{i \in I} \widetilde{\operatorname{ent}}\left(\phi_{i}\right)$.

Proof. If $H$ is a $\phi_{i}$-inert subgroup of $G_{i}$, then it is also a $\phi$-inert subgroup of $G$, therefore $\widetilde{\operatorname{ent}}(\phi) \geq$ $\widetilde{\operatorname{ent}}\left(\phi_{i}\right)$ for all $i \in I$, hence $\widetilde{\operatorname{ent}}(\phi) \geq \sup _{i \in I}\left(\widetilde{\operatorname{ent}}\left(\phi_{i}\right)\right)$.

Conversely, let $H$ be a $\phi$-inert subgroup of $G$. By Lemma 3.10 (b) $H \cap G_{i}$ is a $\phi_{i}$-inert subgroup of $G_{i}$ for every index $i \in I$. To conclude it is enough to prove that there exists an index $i \in I$ such that, for all $n \geq 1$,

$$
\left|T_{n}(\phi, H) / H\right| \leq\left|T_{n}\left(\phi_{i}, H \cap G_{i}\right) /\left(H \cap G_{i}\right)\right| .
$$

In fact, if this occurs, then $\widetilde{\operatorname{ent}}(\phi, H) \leq \widetilde{\operatorname{ent}}\left(\phi_{i}, H \cap G_{i}\right) \leq \widetilde{\operatorname{ent}}\left(\phi_{i}\right)$, therefore $\widetilde{\operatorname{ent}}(\phi, H) \leq \widetilde{\operatorname{ent}}\left(\phi_{i}\right) \leq$ $\sup _{i \in I} \widetilde{\operatorname{ent}}\left(\phi_{i}\right)$, and hence $\widetilde{\operatorname{ent}}(\phi) \leq \sup _{i \in I} \widetilde{\operatorname{ent}}\left(\phi_{i}\right)$. By Proposition 2.2 there exists a finitely generated subset $F$ of $H$ such that for all $n \geq 1$

$$
T_{n}(\phi, H)=H+T_{n}(\phi, F) .
$$

The subset $F$ is contained in some $G_{i}$; therefore, $F \subseteq H \cap G_{i}$ and $T_{n}(\phi, F) \subseteq T_{n}\left(\phi_{i}, H \cap G_{i}\right)$, so

$$
\left(H \cap G_{i}\right)+T_{n}(\phi, F) \subseteq T_{n}\left(\phi_{i}, H \cap G_{i}\right) .
$$

Also the converse inclusion holds true; in fact, $T_{n}\left(\phi_{i}, H \cap G_{i}\right) \subseteq T_{n}(\phi, H) \cap G_{i}$, and since $T_{n}(\phi, F) \subseteq G_{i}$ we can conclude that $T_{n}(\phi, H) \cap G_{i}=\left(H+T_{n}(\phi, F)\right) \cap G_{i}=\left(H \cap G_{i}\right)+T_{n}(\phi, F)$. So we have the equality

$$
T_{n}\left(\phi_{i}, H \cap G_{i}\right)=\left(H \cap G_{i}\right)+T_{n}(\phi, F) .
$$


Therefore $T_{n}(\phi, H) / H \cong\left(H+T_{n}(\phi, F)\right) / H \cong T_{n}(\phi, F) /\left(H \cap T_{n}(\phi, F)\right)$ is isomorphic to a quotient of

$$
T_{n}\left(\phi_{i}, H \cap G_{i}\right) /\left(H \cap G_{i}\right) \cong\left(\left(H \cap G_{i}\right)+T_{n}(\phi, F)\right) /\left(H \cap G_{i}\right) \cong T_{n}(\phi, F) /\left(H \cap G_{i} \cap T_{n}(\phi, F)\right),
$$

hence the desired inequality in $(3.2)$ is proved.

Thanks to Lemma 3.14 we can show now that, given an endomorphism $\phi: G \rightarrow G$ of a torsion-free Abelian group $G$, its intrinsic algebraic entropy equals that of its unique extension to the divisible hull of $G$.

Corollary 3.15. Let $G$ be a torsion-free Abelian group and $\phi$ an endomorphism of $G$. Let $\widetilde{\phi}: D(G) \rightarrow$ $D(G)$ be the unique extension of $\phi$ to the divisible hull. Then

$$
\widetilde{\operatorname{ent}}(\phi)=\widetilde{\operatorname{ent}}(\widetilde{\phi}) \text {. }
$$

Proof. We can identify $G$ with a subgroup of $D(G)$, which is the direct limit of its $\widetilde{\phi}$-invariant subgroups of the form $\frac{1}{n !} G$, with $n$ ranging in $\mathbb{N}_{+}$. Hence, by Lemma 3.14 , we have that $\widetilde{\operatorname{ent}}(\widetilde{\phi})$ is the supremum of the algebraic entropies of the restrictions of $\widetilde{\phi}$ to each $\frac{1}{n !} G$. Furthermore, the groups $\frac{1}{n !} G$ are all isomorphic to $G$ and the restriction of $\widetilde{\phi}$ to $\frac{1}{n !} G$ is conjugated to $\phi$ through this isomorphism. Hence, $\widetilde{\operatorname{ent}}(\phi)=\widetilde{\operatorname{ent}}\left(\tilde{\phi} \Gamma_{(1 / n !) G}\right)$ for all $n \in \mathbb{N}_{+}$, and so $\widetilde{\operatorname{ent}}(\widetilde{\phi})=\sup \left\{\widetilde{\operatorname{ent}}\left(\tilde{\phi} \Gamma_{(1 / n !) G}\right): n \in \mathbb{N}_{+}\right\}=\widetilde{\operatorname{ent}}(\phi)$.

The invariance of the intrinsic algebraic entropy under taking divisible hulls holds only for torsionfree Abelian groups. In fact, consider the torsion group $\mathbb{Z}(p)^{(\mathbb{N})}$, whose divisible hull is $\mathbb{Z}\left(p^{\infty}\right)^{(\mathbb{N})}$. Then $\widetilde{\operatorname{ent}}\left(\beta_{\mathbb{Z}(p)}\right)=\operatorname{ent}\left(\beta_{\mathbb{Z}(p)}\right)=\log p$ while $\widetilde{\operatorname{ent}}\left(\beta_{\mathbb{Z}\left(p^{\infty}\right)}\right)=\infty$ (see Example 3.7 .

Unlike the monotonicity of the function $F \mapsto H(\phi, F)$, its counterpart for ent fails, i.e., $\widetilde{\operatorname{ent}}\left(\phi, H^{\prime}\right) \leq$ $\widetilde{\text { ent }}(\phi, H)$ need not hold for $\phi$-inert subgroups $H^{\prime} \subseteq H$ of $G$ (e.g., take $H=G$ and recall that $\widetilde{\operatorname{ent}}(\phi, G)=0$ ). In item (a) of the next proposition we establish such a monotonicity assuming that the subgroups $H$ and $H^{\prime}$ are "close" in terms of smallness of the quotient $H / H^{\prime}$.

Proposition 3.16. Let $G$ be an Abelian group, $\phi: G \rightarrow G$ an endomorphism and $H^{\prime} \subseteq H$ two $\phi$-inert subgroups of $G$.

(a) If $H / H^{\prime}$ is finitely generated, then $\widetilde{\operatorname{ent}}\left(\phi, H^{\prime}\right) \leq \widetilde{\operatorname{ent}}(\phi, H)$.

(b) If $H$ is finitely generated and $G=T(\phi, H)$, then $\widetilde{\operatorname{ent}}(\phi)=\widetilde{\operatorname{ent}}(\phi, H)$.

Proof. (a) Consider the following exact sequence

$$
0 \rightarrow \frac{H \cap T_{n}\left(\phi, H^{\prime}\right)}{H^{\prime}} \rightarrow \frac{T_{n}\left(\phi, H^{\prime}\right)}{H^{\prime}} \rightarrow \frac{T_{n}(\phi, H)}{H} .
$$

As both $H$ and $H^{\prime}$ are $\phi$-inert, all the groups appearing in the above sequence are finite. This implies that for every $n \in \mathbb{N}_{+}$, the following inequality holds

$$
\log \left|\frac{T_{n}\left(\phi, H^{\prime}\right)}{H^{\prime}}\right|-\log \left|\frac{H \cap T_{n}\left(\phi, H^{\prime}\right)}{H^{\prime}}\right| \leq \log \left|\frac{T_{n}(\phi, H)}{H}\right| .
$$

The subgroup $\left(H / H^{\prime}\right) \cap\left(T\left(\phi, H^{\prime}\right) / H^{\prime}\right)$ of the finitely generated group $H / H^{\prime}$ is finitely generated. Since it is a subgroup of the torsion group $T\left(\phi, H^{\prime}\right) / H^{\prime}$ (see Lemma 2.1(b)), one has $\mid\left(H / H^{\prime}\right) \cap$ $\left(T\left(\phi, H^{\prime}\right) / H^{\prime}\right) \mid<\infty$. For every $n \in \mathbb{N}_{+}$there is an inclusion $\left(H \cap T_{n}\left(\phi, H^{\prime}\right)\right) / H^{\prime} \subseteq\left(H / H^{\prime}\right) \cap$ $\left(T\left(\phi, H^{\prime}\right) / H^{\prime}\right)$, which implies

$$
0 \leq \lim _{n \rightarrow \infty} \frac{\log \left|\left(H \cap T_{n}\left(\phi, H^{\prime}\right)\right) / H^{\prime}\right|}{n} \leq \lim _{n \rightarrow \infty} \frac{\log \left|\left(H / H^{\prime}\right) \cap\left(T\left(\phi, H^{\prime}\right) / H^{\prime}\right)\right|}{n}=0 .
$$


Hence we obtain:

$$
\begin{aligned}
\widetilde{\operatorname{ent}}\left(\phi, H^{\prime}\right) & =\lim _{n \rightarrow \infty} \frac{\log \left|T_{n}\left(\phi, H^{\prime}\right) / H^{\prime}\right|}{n} \\
& =\lim _{n \rightarrow \infty} \frac{\log \left|T_{n}\left(\phi, H^{\prime}\right) / H^{\prime}\right|}{n}-\lim _{n \rightarrow \infty} \frac{\log \left|\left(H \cap T_{n}\left(\phi, H^{\prime}\right)\right) / H^{\prime}\right|}{n} \\
& =\lim _{n \rightarrow \infty} \frac{\log \left|T_{n}\left(\phi, H^{\prime}\right) / H^{\prime}\right|-\log \left|\left(H \cap T_{n}\left(\phi, H^{\prime}\right)\right) / H^{\prime}\right|}{n} \\
& \leq \lim _{n \rightarrow \infty} \frac{\log \left|T_{n}(\phi, H) / H\right|}{n}=\widetilde{\operatorname{ent}}(\phi, H) .
\end{aligned}
$$

(b) Given a $\phi$-inert subgroup $K$ of $G$, we have to prove that $\widetilde{\operatorname{ent}}(\phi, K) \leq \widetilde{\operatorname{ent}}(\phi, H)$. First of all, we can suppose that $H \leq K$, as $\widetilde{\operatorname{ent}}(\phi, K) \leq \widetilde{\operatorname{ent}}(\phi, H+K)$ by item (a) (being $(H+K) / K$ finitely generated). By Proposition 2.2, there exists a finitely generated subgroup $F$ of $G$ such that

$$
\frac{T_{n}(\phi, K)}{K}=\frac{K+T_{n}(\phi, F)}{K},
$$

for every $n \in \mathbb{N}_{+}$. Since $G=T(\phi, H)$ and $F$ is finitely generated, there exists $k \in \mathbb{N}_{+}$such that $F \leq T_{k}(\phi, H)$ and so we obtain that

$$
\frac{T_{n}(\phi, K)}{K}=\frac{K+T_{n}(\phi, F)}{K} \subseteq \frac{K+T_{n+k}(\phi, H)}{K},
$$

for every $n \in \mathbb{N}_{+}$. Since $\left(K+T_{n+k}(\phi, H)\right) / K$ is a quotient of $T_{n+k}(\phi, H) / H$ (as we supposed that $H \leq K)$, the inequality

$$
\left|\frac{T_{n}(\phi, K)}{K}\right| \leq\left|\frac{T_{n+k}(\phi, H)}{H}\right|
$$

holds for every $n \in \mathbb{N}_{+}$. Applying the logarithm, dividing by $n$ and taking the limit we get $\widetilde{\operatorname{ent}}(\phi, K) \leq$ $\widetilde{\operatorname{ent}}\left(\phi, T_{k}(\phi, H)\right)$. To conclude it is enough to apply Lemma 3.11(a).

Part (b) of the above proposition is particularly important as it says that, given a $\phi$-inert finitely generated subgroup $H$ of $G$, the quantity $\widetilde{\operatorname{ent}}(\phi, H)$ does not depend on $H$ but only on $T(\phi, H)$, that is, for any other finitely generated a $\phi$-inert subgroup $H^{\prime}$ of $G$ with $T(\phi, H)=T\left(\phi, H^{\prime}\right)$, one has $\widetilde{\operatorname{ent}}(\phi, H)=\widetilde{\operatorname{ent}}\left(\phi, H^{\prime}\right)=\widetilde{\operatorname{ent}}\left(\phi \uparrow_{T(\phi, H)}\right)$.

\section{Relationship with the Algebraic Yuzvinski Formula}

For $m \in \mathbb{N}_{+}$, an endomorphism $\phi: \mathbb{Q}^{m} \rightarrow \mathbb{Q}^{m}$ is described by an $m \times m$ matrix with coefficients in $\mathbb{Q}$, which has its monic characteristic polynomial $f_{\phi}(X) \in \mathbb{Q}[X]$; we say that $f_{\phi}(X)$ is the characteristic polynomial of $\phi$ over $\mathbb{Q}$. Let $s$ be the least positive integer such that $s f_{\phi}(X) \in \mathbb{Z}[X]$ (i.e., $s$ is the least common multiple of the denominators of the coefficients of $\left.f_{\phi}(X)\right)$. We call $p_{\phi}(X)=s f_{\phi}(X)$ the characteristic polynomial of $\phi$ over $\mathbb{Z}$. The roots of $f_{\phi}(X)$ (which are the same of $\left.p_{\phi}(X)\right)$ are the eigenvalues $\left\{\lambda_{i}: i=1, \ldots, m\right\}$ of $\phi$.

Recall that the quantity

$$
\mathrm{m}\left(p_{\phi}(X)\right)=\log s+\sum_{\left|\lambda_{i}\right|>1} \log \left|\lambda_{i}\right|
$$

is the (logarithmic) Mahler measure of $p_{\phi}(X)$ (see [13], [14] and [12] for more details). The Mahler measure of $\phi$ is $\mathrm{m}(\phi)=\mathrm{m}\left(p_{\phi}(X)\right)$.

The main result of the recent paper [12] is the following formula concerning the algebraic entropy $h$ of an endomorphism $\phi$ of $\mathbb{Q}^{m}$. 
Theorem 4.1 (Algebraic Yuzvinski Formula). Let $m$ be a positive integer and $\phi: \mathbb{Q}^{m} \rightarrow \mathbb{Q}^{m}$ an endomorphism. Then

$$
h(\phi)=\mathrm{m}(\phi) .
$$

While the eigenvalues appearing in the Mahler measure have a clear dynamical meaning in terms of "expansiveness" of $\phi$, the appearance of $\log s$ is somehow surprising. The main result of this section is the following algebraic interpretation of this mysterious term, generalizing the 1-dimensional case considered in Example $3.4(2)$.

Theorem 4.2. Let $m$ be a positive integer, $\phi: \mathbb{Q}^{m} \rightarrow \mathbb{Q}^{m}$ an endomorphism and let $s$ be the positive leading coefficient of the characteristic polynomial of $\phi$ over $\mathbb{Z}$. Then

$$
\log s=\widetilde{\operatorname{ent}}(\phi) .
$$

Actually, we prove that $\log s=\widetilde{\operatorname{ent}}\left(\phi, \mathbb{Z}^{m}\right)$, from which Theorem 4.2 follows. In fact, $\widetilde{\operatorname{ent}}(\phi)=$ $\widetilde{\operatorname{ent}}\left(\phi \uparrow_{T\left(\phi, \mathbb{Z}^{m}\right)}\right)$ by Corollary 3.15 and $\widetilde{\operatorname{ent}}\left(\phi \uparrow_{T\left(\phi, \mathbb{Z}^{m}\right)}\right)=\widetilde{\operatorname{ent}}\left(\phi, \mathbb{Z}^{m}\right)$ by Proposition 3.16(b). The proof of the equality $\log s=\widetilde{\operatorname{ent}}\left(\phi, \mathbb{Z}^{m}\right)$, deferred to 4.2 , will follow as a consequence of some results from [12.

A consequence of Theorems 4.1 and 4.2 is that the difference between the algebraic entropy $h$ and the intrinsic algebraic entropy of an endomorphism $\phi$ of a torsion-free Abelian group $G$ of finite rank, depends only on the eigenvalues of the endomorphism, that is

$$
h(\phi)-\widetilde{\operatorname{ent}}(\phi)=\sum_{\left|\lambda_{i}\right|>1} \log \left|\lambda_{i}\right| .
$$

\subsection{Algebraic entropy for locally compact Abelian groups}

For a LCA group $G$ denote by $\mathcal{C}(G)$ the family of all compact neighborhoods of 0 in $G$ and note that $\mathcal{C}(G)$ contains the family $\mathcal{B}(G)$ of all open compact subgroups of $G$. Let $\mu$ be a Haar measure on $G$.

For a continuous endomorphism $\phi: G \rightarrow G$, the algebraic entropy of $\phi$ with respect to $C \in \mathcal{C}(G)$ is

$$
H(\phi, C)=\limsup _{n \rightarrow \infty} \frac{\log \mu\left(T_{n}(\phi, C)\right)}{n},
$$

and the algebraic entropy of $\phi$ is

$$
h(\phi)=\sup \{H(\phi, C): C \in \mathcal{C}(G)\} .
$$

Our strategy for the proof of Theorem 4.2 is to find a connection between $h$ and ent, which holds for continuous endomorphisms of totally disconnected LCA groups, implying in particular that $h\left(\phi_{p}\right)=\widetilde{\operatorname{ent}}\left(\phi_{p}\right)$ for all $p \in \mathbb{P}$ (Theorem 4.3 and Corollary 4.7). Then, we show that $\widetilde{\operatorname{ent}}\left(\phi, \mathbb{Z}^{m}\right)=$ $\sum_{p \in \mathbb{P}} \widetilde{\operatorname{ent}}\left(\phi_{p}, \mathbb{J}_{p}^{m}\right)$ (Lemma 4.8.

Theorem 4.3. Let $G$ be a $L C A$ group and $\phi: G \rightarrow G$ be a continuous endomorphism. Then $\mathcal{B}(G) \subseteq$ $\mathcal{I}_{\phi}(G)$. Furthermore,

$$
H(\phi, U)=\widetilde{\operatorname{ent}}(\phi, U)
$$

for all $U \in \mathcal{B}(G)$.

Proof. Let $U \in \mathcal{B}(G)$. Then the subgroup $U$ of the compact subgroup $T_{n}(\phi, U)$ of $G$ is open, so $T_{n}(\phi, U) / U$ is finite. Consider now a Haar measure $\mu$ on $G$. Since $T_{n}(\phi, U)$ is the disjoint union of $\left|T_{n}(\phi, U) / U\right|$-many cosets of the form $x+U$, with $x \in T_{n}(\phi, U)$, one has

$$
\mu\left(T_{n}(\phi, U)\right)=\mu(U) \cdot\left|T_{n}(\phi, U) / U\right| .
$$


Moreover, $0<\mu(U)<\infty$, as $U$ is open and compact; in particular, $\lim _{n \rightarrow \infty} \frac{\log \mu(U)}{n}=0$. Therefore,

$$
H(\phi, U)=\lim _{n \rightarrow \infty} \frac{\log \mu(U)+\log \left|T_{n}(\phi, U) / U\right|}{n}=\lim _{n \rightarrow \infty} \frac{\log \left|T_{n}(\phi, U) / U\right|}{n}=\widetilde{\operatorname{ent}}(\phi, U),
$$

and this concludes the proof.

Remark 4.4. In the sequel $G$ will be a totally disconnected locally compact group $G$. Recall that for such a group $G$ the family $\mathcal{B}(G)$ is a local base at the neutral element, according to a theorem of van Dantzig [23].

(a) If the group $G$ is discrete, then the open compact subgroups of $G$ are necessarily finite, so the equality 4.1 in Theorem 4.3 obviously holds true.

(b) In case the totally disconnected group $G$ is covered by compact subgroups, then by taking the supremum in 4.1), whit $U$ running over $\mathcal{B}(G)$, one gets the algebraic entropy $h(\phi)$ on the left hand side. On the right hand side one gets a quantity that is $\leq \widetilde{\operatorname{ent}}(\phi)$. Therefore,

$$
h(\phi) \leq \widetilde{\operatorname{ent}}(\phi) .
$$

Furthermore, if $G$ is discrete, then the current hypothesis entails that $G$ is torsion, in which case the opposite inequality in 4.2 is proved in Proposition 3.6 .

The next example shows that the inequality in $(4.2)$ can be strict in the non-discrete case.

Example 4.5. Let $p$ be a prime. If $\xi \in \mathbb{J}_{p}$ is a $p$-adic integer transcendental over $\mathbb{Z}$, then the multiplication $\phi_{\xi}$ by $\xi$ in $\mathbb{Q}_{p}$ (equipped with the $p$-adic topology) has algebraic entropy zero as proved in [7, Example 5.6.5], while its intrinsic algebraic entropy is $\infty$. Indeed, $\phi_{\xi}$ restricted to the subgroup $\mathbb{Z}[\xi]$ is conjugated to the right Bernoulli shift and so it suffices to apply Lemma 3.9. Lemma 3.10(a) and Example 3.7 .

\subsection{Proof of Theorem 4.2}

The following Proposition 4.6 is an easy consequence of two results of [12], that is, Theorem 3.8 and the Corollary stated in the Introduction. In order to state this result we need to recall some notations.

Let $m$ be a positive integer and $\phi: \mathbb{Q}^{m} \rightarrow \mathbb{Q}^{m}$ an endomorphism. For every $p \in \mathbb{P}, \phi$ induces an endomorphism $\phi_{p}: \mathbb{Q}_{p}^{m} \rightarrow \mathbb{Q}_{p}^{m}$ just extending the scalars, that is,

$$
\phi_{p}=\phi \otimes_{\mathbb{Q}} i d_{\mathbb{Q}_{p}} .
$$

Proposition 4.6. [12] Let $m$ be a positive integer, $\phi: \mathbb{Q}^{m} \rightarrow \mathbb{Q}^{m}$ an endomorphism and $p_{\phi}(X)=$ $s X^{m}+a_{1} X^{m-1}+\ldots+a_{m}$ the characteristic polynomial over $\mathbb{Z}$ of $\phi$. Then

$$
h\left(\phi_{p}\right)=H\left(\phi_{p}, \mathrm{~J}_{p}^{m}\right)=\log |1 / s|_{p} .
$$

Using this proposition we can compute the algebraic entropy $h$ of the various $\phi_{p}$. From this result and from Theorem 4.3 we derive the following consequence.

Corollary 4.7. Let $m$ be a positive integer, $\phi: \mathbb{Q}^{m} \rightarrow \mathbb{Q}^{m}$ an endomorphism and, for a fixed prime $p$, let $\phi_{p}: \mathbb{Q}_{p}^{m} \rightarrow \mathbb{Q}_{p}^{m}$ the induced endomorphism. Then

$$
h\left(\phi_{p}\right)=\lim _{n \rightarrow \infty} \frac{\log \left|T_{n}\left(\phi_{p}, \mathbb{J}_{p}^{m}\right) / \mathbb{J}_{p}^{m}\right|}{n}=\widetilde{\operatorname{ent}}\left(\phi_{p}, \mathbb{J}_{p}^{m}\right) .
$$


Proof. By Proposition 4.6, we have that $h\left(\phi_{p}\right)=H\left(\phi_{p}, \mathbb{J}_{p}^{m}\right)$. To conclude, apply Theorem 4.3 with $G=\mathbb{Q}_{p}^{m}, U=\mathbb{J}_{p}^{m}$ and $\phi=\phi_{p}$.

The next lemma is the last step for the proof of Theorem 4.2. Remember that $\mathbb{J}_{p}$ is a valuation domain, furthermore it is flat when considered as a module over $\mathbb{Z}$. The field of quotients of $\mathbb{J}_{p}$ is the field $\mathbb{Q}_{p}$ of $p$-adic numbers.

Lemma 4.8. For every $n \in \mathbb{N}_{+}$, we have that

$$
\left|T_{n}\left(\phi, \mathbb{Z}^{m}\right) / \mathbb{Z}^{m}\right|=\prod_{p \in \mathbb{P}}\left|T_{n}\left(\phi_{p}, \mathbb{J}_{p}^{m}\right) / \mathbb{J}_{p}^{m}\right|,
$$

where on the right hand side only finitely many factors are $>1$.

Proof. In order to simplify the notation, we set $Q_{n}=T_{n}\left(\phi, \mathbb{Z}^{m}\right) / \mathbb{Z}^{m}$. Consider the exact sequence

$$
0 \rightarrow \mathbb{Z}^{m} \rightarrow T_{n}\left(\phi, \mathbb{Z}^{m}\right) \rightarrow Q_{n} \rightarrow 0 .
$$

Let $p \in \mathbb{P}$; as we said $\mathbb{J}_{p}$ is a flat $\mathbb{Z}$-module. In particular the following sequence of $\mathbb{J}_{p}$-modules is exact

$$
0 \rightarrow \mathbb{Z}^{m} \otimes_{\mathbb{Z}} \mathbb{J}_{p} \rightarrow T_{n}\left(\phi, \mathbb{Z}^{m}\right) \otimes_{\mathbb{Z}} \mathbb{J}_{p} \rightarrow Q_{n} \otimes_{\mathbb{Z}} \mathbb{J}_{p} \rightarrow 0 .
$$

Since $\mathbb{Q} \otimes_{\mathbb{Z}} \mathbb{J}_{p} \cong \mathbb{Q}_{p}$, we can identify $\mathbb{Z}^{m} \otimes_{\mathbb{Z}} \mathbb{J}_{p}$ and $T_{n}\left(\phi, \mathbb{Z}^{m}\right) \otimes_{\mathbb{Z}} \mathbb{J}_{p}$ with $\mathbb{J}_{p}$-submodules of $\mathbb{Q}_{p}^{m}$. Fix the canonical base $\left\{e_{1}, \ldots, e_{m}\right\}$ of $\mathbb{Q}^{m}$ as a $\mathbb{Q}$-vector space. Then $\left\{e_{1} \otimes 1_{\mathbb{J}_{p}}, \ldots, e_{m} \otimes 1_{\mathbb{J}_{p}}\right\}$ is a base of $\mathbb{Q}_{p}^{m}$ as a $\mathbb{Q}_{p}$-vector space.

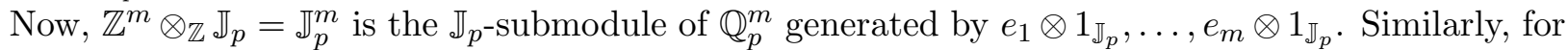
every $k \in \mathbb{N}, \phi^{k}\left(\mathbb{Z}^{m}\right)$ is the subgroup of $\mathbb{Q}^{m}$ generated by $\phi^{k}\left(e_{1}\right), \ldots, \phi^{k}\left(e_{m}\right)$. This shows that

$$
\begin{aligned}
\left(\phi^{k} \mathbb{Z}^{m}\right) \otimes_{\mathbb{Z}} \mathbb{J}_{p} & =\left(\phi^{k}\left(e_{1}\right) \otimes 1_{\mathbb{J}_{p}}\right) \mathbb{J}_{p}+\cdots+\left(\phi^{k}\left(e_{m}\right) \otimes 1_{\mathbb{J}_{p}}\right) \mathbb{J}_{p} \\
& =\left(\phi_{p}^{k}\left(e_{1} \otimes 1_{\mathbb{J}_{p}}\right)\right) \mathbb{J}_{p}+\cdots+\left(\phi_{p}^{k}\left(e_{m} \otimes 1_{\mathbb{J}_{p}}\right)\right) \mathbb{J}_{p}=\phi_{p}^{k}\left(\mathbb{J}_{p}^{m}\right) .
\end{aligned}
$$

In a similar way one can show that $T_{n}\left(\phi, \mathbb{Z}^{m}\right) \otimes_{\mathbb{Z}} \mathbb{J}_{p}=T_{n}\left(\phi_{p}, \mathbb{J}_{p}^{m}\right)$ for every $n \in \mathbb{N}_{+}$.

Since $\mathbb{J}_{p}$ is $q$-divisible for every prime $q \neq p$, we have that $Q_{n} \otimes_{\mathbb{Z}} \mathbb{J}_{p}$ is isomorphic to the $p$-component $t_{p}\left(Q_{n}\right)$ of $Q_{n}$. Hence equation 4.3 becomes

$$
0 \rightarrow \mathbb{J}_{p}^{m} \rightarrow T_{n}\left(\phi_{p}, \mathbb{J}_{p}^{m}\right) \rightarrow t_{p}\left(Q_{n}\right) \rightarrow 0 .
$$

It follows that

$$
\left|T_{n}\left(\phi, \mathbb{Z}^{m}\right) / \mathbb{Z}^{m}\right|=\left|Q_{n}\right|=\left|\bigoplus_{p \in \mathbb{P}} t_{p}\left(Q_{n}\right)\right|=\prod_{p \in \mathbb{P}}\left|t_{p}\left(Q_{n}\right)\right|=\prod_{p \in \mathbb{P}}\left|T_{n}\left(\phi, \mathbb{J}_{p}^{m}\right) / \mathbb{J}_{p}^{m}\right|,
$$

as desired. Since $Q_{n}$ is finite, the family of primes $p$ such that $t_{p}\left(Q_{n}\right)$ is non-zero is a finite subset of $\mathbb{P}$. In particular, in the product appearing on the right hand side of the statement, only a finite number of terms are $>1$.

In the following remark we give another explication to the last claim in Lemma 4.8.

Remark 4.9. Let $A=\left(a_{i j}\right)$ be the matrix of $\phi$ with respect to the canonical base of $\mathbb{Q}^{m}$. The coefficients of $A$ are all rational numbers; for every $i, j=1, \ldots, m$ we let $a_{i j}=m_{i j} / d_{i j}$ with $m_{i j}, d_{i j} \in \mathbb{Z}$ and $\left(m_{i j}, d_{i j}\right)=1$. Suppose first that all the coefficients of $A$ are integers, that is, $d_{i j}=1$ for every $i, j$. Then the subgroup $\mathbb{Z}^{m}$ of $\mathbb{Q}^{m}$ is $\phi$-invariant and so $T_{n}\left(\phi, \mathbb{Z}^{m}\right)=\mathbb{Z}^{m}$ for every $n \in \mathbb{N}_{+}$. Hence $\left|T_{n}\left(\phi, \mathbb{Z}^{m}\right) / \mathbb{Z}^{m}\right|=\left|\mathbb{Z}^{m} / \mathbb{Z}^{m}\right|=1$. (In this case we have log $s=0$.) Similarly, suppose that $p \in \mathbb{P}$ does 
not divide any $d_{i j}$ with $i, j=1, \ldots, m$. Denote by $\mathbb{Z}_{p}$ the localization of $\mathbb{Z}$ at the prime ideal $p \mathbb{Z}$. It is clear in this situation that $\mathbb{Z}_{p}^{m}$ is $\phi$-invariant. In particular, $\left|T_{n}\left(\phi, \mathbb{Z}_{p}^{m}\right) / \mathbb{Z}_{p}^{m}\right|=\left|\mathbb{Z}_{p}^{m} / \mathbb{Z}_{p}^{m}\right|=1$. Since $\mathbb{Z}_{p} \otimes_{\mathbb{Z}} \mathbb{J}_{p}=\mathbb{Z} \otimes_{\mathbb{Z}} \mathbb{J}_{p}$, if $\mathbb{Z}_{p}^{m}$ is $\phi$-invariant in $\mathbb{Q}^{m}$ then $\mathbb{J}_{p}^{m}$ is $\phi_{p}$-invariant in $\mathbb{Q}_{p}^{m}$. In particular, in the above situation, $\left|T_{n}\left(\phi_{p}, \mathbb{J}_{p}^{m}\right) / \mathbb{J}_{p}^{m}\right|=\left|\mathbb{J}_{p}^{m} / \mathbb{J}_{p}^{m}\right|=1$.

Thus we have the following inclusion

$$
\left\{p \in \mathbb{P}:\left|T_{n}\left(\phi_{p}, \mathbb{J}_{p}^{m}\right) / \mathbb{J}_{p}^{m}\right|>1\right\} \subseteq\left\{p \in \mathbb{P}: p \mid d_{i j} \text { for some } i, j=1, \ldots, m\right\} .
$$

We are now in position to demonstrate Theorem 4.2 .

Proof of Theorem 4.2. By Lemma 4.8 we get

$$
\widetilde{\operatorname{ent}}\left(\phi, \mathbb{Z}^{m}\right)=\sum_{p \in \mathbb{P}} \widetilde{\operatorname{ent}}\left(\phi_{p}, \mathbb{J}_{p}^{m}\right) .
$$

Now, Corollary 4.7 and Proposition 4.6 give

$$
\widetilde{\operatorname{ent}}\left(\phi_{p}, \mathbb{J}_{p}^{m}\right)=h\left(\phi_{p}\right)=\log |1 / s|_{p}
$$

Since $s=\prod_{p \in \mathbb{P}}|1 / s|_{p}$, we can conclude with 4.4 that $\widetilde{\operatorname{ent}}\left(\phi, \mathbb{Z}^{m}\right)=\log s$, as desired.

\section{The Addition Theorem}

We start this section by recalling some notions concerning real-valued invariants of a category of modules. Let $R$ be a ring and $L: \operatorname{Mod}(R) \rightarrow \mathbb{R}^{*}$ a map; we say that $L$ is an invariant if $L(0)=0$ and $L(M)=L\left(M^{\prime}\right)$ whenever $M \cong M^{\prime}$; and that $L$ is an additive invariant if, in addition, $L(M)=$ $L\left(M^{\prime}\right)+L\left(M^{\prime \prime}\right)$ for any short exact sequence $0 \rightarrow M^{\prime} \rightarrow M \rightarrow M^{\prime \prime} \rightarrow 0$ in $\operatorname{Mod}(R)$.

Clearly, if $L$ is an additive invariant of $\operatorname{Mod}(R)$ and $M$ is an $R$-module, then, given a finite chain of submodules $0=M_{0} \subseteq M_{1} \subseteq \ldots \subseteq M_{n}=M$, we have that $L(M)=\sum_{i=1}^{n} L\left(M_{i} / M_{i-1}\right)$.

An invariant $L: \operatorname{Mod}(R) \rightarrow \mathbb{R}^{*}$ is upper continuous if

$$
L(M)=\sup \{L(F): F \in \mathfrak{F}(M)\},
$$

for every $M \in \operatorname{Mod}(R)$, where $\mathfrak{F}(M)$ denotes the family of all the finitely generated submodules of $M$. If $L$ is both additive and upper continuous, then $L$ is called a length function. Length functions have been introduced by Northcott-Reufel [15] and were investigated also by Vámos in [21] and [22].

The following result is contained in [22, Proposition 8].

Lemma 5.1. 22] Given a ring $R$ and an additive invariant $L: \operatorname{Mod}(R) \rightarrow \mathbb{R}^{*}$, the following conditions are equivalent:

(a) L is upper continuous;

(b) for every $R$-module $M$ and every directed system $\left\{M_{i}: i \in I\right\}$ of submodules of $M$ such that $\bigcup_{i \in I} M_{i}=M$, we have $L(M)=\sup \left\{L\left(M_{i}\right): i \in I\right\}$;

(c) for every R-module $M$ and every totally ordered set $\left\{M_{i}: i \in I\right\}$ of submodules of $M$ such that $\bigcup_{i \in I} M_{i}=M$, we have $L(M)=\sup \left\{L\left(M_{i}\right): i \in I\right\}$. 
The meaning of Lemma 5.1 is that any additive invariant which is continuous on direct limits is completely determined by the values it assumes on finitely generated modules. This becomes even more convenient over Noetherian rings (so also for Abelian groups), where the finitely generated modules form a Serre subclass of $\operatorname{Mod}(\mathrm{R})$. In the following proposition we see that an upper continuous invariant is a length function if and only if it is additive on finitely generated modules. This fact will be used in demonstrating the Addition Theorem.

Proposition 5.2. Let $R$ be a Noetherian ring and $L: \operatorname{Mod}(\mathrm{R}) \rightarrow \mathbb{R}^{*}$ be an upper continuous invariant. If $L\left(F_{2}\right)=L\left(F_{1}\right)+L\left(F_{3}\right)$ for every short exact sequence $0 \rightarrow F_{1} \rightarrow F_{2} \rightarrow F_{3} \rightarrow 0$ of finitely generated $R$-modules, then $L$ is additive, hence a length function.

Proof. Since $L$ is upper continuous by hypothesis, it remains only to check additivity. To this end, let $N$ and $M$ be $R$-modules such that $N \subseteq M$, and choose $F \in \mathfrak{F}(M)$; so $F \cap N \in \mathfrak{F}(N)$ since $R$ is Noetherian, and $(F+N) / N \in \mathfrak{F}(M / N)$. By the additivity of $L$ on finitely generated modules, we obtain $L(F)=L(F \cap N)+L((F+N) / N)$ which proves that $L(M) \leq L(N)+L(M / N)$ by the arbitrariness of $F$.

On the other hand, let $F_{1} \in \mathfrak{F}(N)$ and $\bar{F}_{2} \in \mathfrak{F}(M / N)$. Choose a submodule $F_{2} \in \mathfrak{F}(M)$ such that $\left(F_{2}+N\right) / N=\bar{F}_{2}$ and set $F=F_{1}+F_{2}$. Then

$$
L(F)=L(F \cap N)+L((F+N) / N) \geq L\left(F_{1}\right)+L\left(\bar{F}_{2}\right),
$$

since $\bar{F}_{2}=(F+N) / N$ (being $F_{1}$ contained in $\left.N\right)$ and $F_{1} \subseteq F \cap N$. This gives $L(M) \geq L(N)+L(M / N)$, concluding the proof.

Usually a mathematical notion of entropy (no matter if topological, algebraic, measure-theoretic) is defined as a map from the set of endomorphisms of a fixed object in a category $\mathfrak{C}$ to $\mathbb{R}^{*}$. This point of view can be made categorical as follows (see [6] and [20]).

Given a category $\mathfrak{C}$, let Flow $(\mathfrak{C})$ denote the category of flows of $\mathfrak{C}$, whose objects are the pairs $(G, \phi)$ where $G$ is an object of $\mathfrak{C}$ and $\phi: G \rightarrow G$ is an endomorphism. A morphism $\left(G_{1}, \phi\right) \rightarrow\left(G_{2}, \psi\right)$ in $\operatorname{Flow}(\mathfrak{C})$ is a commutative square

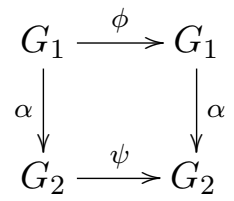

where $\alpha: G_{1} \rightarrow G_{2}$ is a morphism in $\mathfrak{C}$. Abusing notation, we shall denote by $\alpha$ also the morphism $\left(G_{1}, \phi_{1}\right) \rightarrow\left(G_{2}, \phi_{2}\right)$ in $\operatorname{Flow}(\mathfrak{C})$.

When the category $\mathfrak{C}$ is the category $\operatorname{Mod}(R)$, there is a nice description of the category of flows; in fact, the category Flow $(\operatorname{Mod}(\mathrm{R}))$ is canonically isomorphic to $\operatorname{Mod}(R[X])$. The canonical isomorphism is given by $(G, \phi) \mapsto G_{\phi} \in \operatorname{Mod}(R[X])$, where $G_{\phi}$ as an $R$-module is just $G$ and $X$ acts on $G$ via $\phi$, that is

$$
\left(r_{0}+r_{1} X+\cdots+r_{n} X^{n}\right) \cdot g=r_{0} g+r_{1} \phi(g)+\cdots+r_{n} \phi^{n}(g)
$$

for all $g \in G$ and $\left(r_{0}+r_{1} X+\cdots+r_{n} X^{n}\right) \in R[X]$. Also a morphism in Flow $(\operatorname{Mod}(R))$ is easily seen to commute with the action of $X$ and thus it becomes an $R[X]$-module homomorphism.

In what follows we will identify the categories Flow $(\operatorname{Mod}(R))$ and $\operatorname{Mod}(R[X])$, denoting by $G_{\phi}$ a generic object.

Let $G_{\phi}$ be an $R[X]$-module. An $R[X]$-submodule of $G_{\phi}$ is nothing else but a $\phi$-invariant submodule of $G$. Similarly, a quotient of $G_{\phi}$ is just a quotient of $G$ such that the kernel of the natural projection 
is $\phi$-invariant. Recall that a segment of $G_{\phi}$ is any submodule of a quotient of $G_{\phi}$. We shall sometimes abuse notation, denoting by $H_{\phi}$ a segment of $G_{\phi}$ with $X$ acting as the endomorphism induced on $H$ by $\phi$.

Notice that it is possible to consider the intrinsic algebraic entropy as an invariant of $\operatorname{Mod}(\mathbb{Z}[X])$. Indeed, we let

$$
\widetilde{\text { ent }}: \operatorname{Mod}(\mathbb{Z}[X]) \rightarrow \mathbb{R}^{*}, G_{\phi} \longmapsto \widetilde{\operatorname{ent}}(\phi) .
$$

By Lemma 3.9 ent is an invariant and by Lemma 3.14 ent is also upper continuous.

The remaining part of this section is devoted to prove the Addition Theorem for ent, which says that, given a $\mathbb{Z}[X]$-module $G_{\phi}$ and a $\mathbb{Z}[X]$-submodule $H_{\phi}$ of $G_{\phi}$, then $\widetilde{\operatorname{ent}}\left(G_{\phi}\right)=\widetilde{\operatorname{ent}}\left(H_{\phi}\right)+$ $\widetilde{\operatorname{ent}}\left((G / H)_{\phi}\right)$.

The next two results give the proof of the Addition Theorem in the very particular case of a divisible torsion-free Abelian group of finite rank with a pure $\phi$-invariant subgroup. The proof of the next lemma is analogous to that of [6, Proposition 4.2] in the case of $h$.

Lemma 5.3. Let $m$ be a positive integer and $\phi: \mathbb{Q}^{m} \rightarrow \mathbb{Q}^{m}$ an endomorphism. Suppose that $K \cong \mathbb{Q}^{k}$, for some $k \leq m$, is a $\phi$-invariant $\mathbb{Q}$-vector subspace of $\mathbb{Q}^{m}$. Then

$$
\widetilde{\operatorname{ent}}(\phi)=\widetilde{\operatorname{ent}}\left(\phi \uparrow_{K}\right)+\widetilde{\operatorname{ent}}(\bar{\phi}) \text {, }
$$

where $\bar{\phi}: \mathbb{Q}^{m} / K \rightarrow \mathbb{Q}^{m} / K$ is the endomorphism induced by $\phi$.

Proof. Choose a basis $\mathcal{B}=\left\{v_{1}, \ldots, v_{k}, v_{k+1}, \ldots, v_{m}\right\}$ of $\mathbb{Q}^{m}$ such that $\mathcal{B}_{K}=\left\{v_{1}, \ldots, v_{k}\right\}$ is a basis of $K$; the matrix of $\phi$ with respect to $\mathcal{B}$ has the following block-wise form:

$$
A=\left(\begin{array}{cc}
A_{1} & B \\
0 & A_{2}
\end{array}\right),
$$

where $A_{1}$ is the matrix of $\phi \uparrow_{K}$ with respect to $\mathcal{B}_{K}$. Let $\pi: \mathbb{Q}^{m} \rightarrow \mathbb{Q}^{m} / K$ be the canonical projection and let $\overline{\mathcal{B}}=\left\{\pi\left(v_{k+1}\right), \ldots, \pi\left(v_{m}\right)\right\}$, which is a basis of $\mathbb{Q}^{m} / K$. Then $A_{2}$ is the matrix of $\bar{\phi}$ with respect to $\overline{\mathcal{B}}$. Let $\alpha_{1}, \ldots, \alpha_{k}$ be the eigenvalues of $A_{1}$ and let $\alpha_{k+1}, \ldots, \alpha_{m}$ be the eigenvalues of $A_{2}$.

Let $f_{\phi}(X), f_{1}(X), f_{2}(X) \in \mathbb{Q}[X]$ be the characteristic polynomials of $A, A_{1}, A_{2}$ respectively. Then $f_{\phi}(X)=f_{1}(X) \cdot f_{2}(X)$. Let $s_{1}$ and $s_{2}$ be the least common multiples of the denominators of the coefficients of $f_{1}$ and $f_{2}$ respectively. This means that $p_{1}(X)=s_{1} f_{1}(X)$ and $p_{2}(X)=s_{2} f_{2}(X) \in \mathbb{Z}[X]$ are primitive. By Gauss Lemma, $p_{\phi}(X)=p_{1}(X) \cdot p_{2}(X)$ is primitive and so, setting $s=s_{1} s_{2}$, the polynomial $p_{\phi}(X)=s f_{\phi}(X) \in \mathbb{Z}[X]$ is primitive. Now Theorem 4.2 applied to $\phi, \phi \uparrow_{K}$ and $\bar{\phi}$ gives $\widetilde{\operatorname{ent}}(\phi)=\log s=\log s_{1}+\log s_{2}=\widetilde{\operatorname{ent}}\left(\phi \uparrow_{K}\right)+\widetilde{\operatorname{ent}}(\bar{\phi})$.

Proposition 5.4. Let $G$ be a torsion-free Abelian group of finite rank and $\phi: G \rightarrow G$ an endomorphism. If $H$ is a $\phi$-invariant pure subgroup of $G$ then

$$
\widetilde{\operatorname{ent}}(\phi)=\widetilde{\operatorname{ent}}\left(\phi \uparrow_{H}\right)+\widetilde{\operatorname{ent}}(\bar{\phi}),
$$

where $\bar{\phi}: G / H \rightarrow G / H$ is the morphism induced by $\phi$.

Proof. As $H$ is pure in $G$ we have that $G / H$ is torsion-free. Furthermore, the following exact sequence

$$
0 \rightarrow H \rightarrow G \rightarrow G / H \rightarrow 0
$$

is sent to the following short exact sequence tensorizing by $\mathbb{Q}$ :

$$
0 \rightarrow D(H) \rightarrow D(G) \rightarrow D(G / H) \rightarrow 0 .
$$

Now, to conclude the proof it is enough to use the fact that the intrinsic algebraic entropy is preserved by taking divisible hulls of torsion-free Abelian groups by Corollary 3.15, and that it is additive on sequences of rational vector spaces by Lemma 5.3 . 
Our next goal is to prove the Addition Theorem in the particular case when $G$ is a finite-rank torsion-free Abelian group that is the trajectory of a finitely generated subgroup, and $H$ is a $\phi$ invariant subgroup of $G$ of maximal rank.

Proposition 5.5. Let $G$ be a torsion-free Abelian group of finite rank $k \in \mathbb{N}_{+}, \phi: G \rightarrow G$ an endomorphism and suppose there exists a finitely generated subgroup $F$ of $G$ such that $G=T(\phi, F)$. Let $H$ be a $\phi$-invariant subgroup of $G$ such that $G / H$ is torsion. Then:

(a) $G / H$ is finite;

(b) $\widetilde{\operatorname{ent}}(\phi)=\widetilde{\operatorname{ent}}\left(\phi \uparrow_{H}\right)$ and $\widetilde{\operatorname{ent}}(\bar{\phi})=0$, where $\bar{\phi}$ is the endomorphism induced by $\phi$ on $G / H$.

Proof. There exist finitely many prime numbers $\left\{p_{1}, \ldots, p_{h}\right\}$ such that, identifying $G$ with a subgroup of its divisible hull $D(G)=\mathbb{Q}^{k}$, we have $G \subseteq \mathbb{Z}\left[1 / p_{1}, \ldots, 1 / p_{h}\right]^{k}$, where $\mathbb{Z}\left[1 / p_{1}, \ldots, 1 / p_{h}\right]=R$ is the subring of $\mathbb{Q}$ generated by the fractions $1 / p_{i}$ with $1 \leq i \leq h$. Indeed, since $F$ is finitely generated, we can suppose $F \cong \mathbb{Z}^{m} \subseteq \mathbb{Z}^{k}$ for some $m \leq k$. Let $\left\{p_{1}, \ldots, p_{h}\right\}$ be the family of primes dividing the coefficients of the rational matrix associated to the extension $\tilde{\phi}$ of $\phi$ to $D(G)$. Then clearly $\phi^{n}(F) \subseteq R^{k}$ for every $n \in \mathbb{N}$, hence $G=T(\phi, F) \subseteq R^{k}$.

(a) As $(G / H)_{\phi}$ is a finitely generated $\mathbb{Z}[X]$-module, any positive integer annihilating its generators annihilates $G / H$, which is therefore bounded. A bounded Abelian group with finite socle is finite, so it is enough to prove that $G / H$ has finite socle. Since the class of torsion Abelian groups with finite socle is closed under taking subgroups, quotients and finite direct sums, recalling that $G / H$ is contained into $R^{k} / H$, and noting that $R^{k} / H$ is a quotient of $R / m_{1} \mathbb{Z} \oplus \ldots \oplus R / m_{k} \mathbb{Z}$ for suitable non-zero integers $m_{1}, \ldots, m_{k}$, the conclusion comes from the fact that the socle of $R / n \mathbb{Z}$ is finite for all $n \in \mathbb{Z}$, because $R / n \mathbb{Z}$ is a finite direct sum of cocyclic groups.

(b) As $G$ and $H$ have the same rank, $D(G)=D(H)$ and so it follows by Corollary 3.15 that $\widetilde{\operatorname{ent}}(\phi)=\widetilde{\operatorname{ent}}\left(\phi \uparrow_{H}\right)$. Furthermore, $G / H$ is finite by item $(\mathrm{a})$ and so $\widetilde{\operatorname{ent}}(\bar{\phi})=0$.

We pass now to examine Abelian groups which fail to be torsion-free, but which still are trajectories of finitely generated subgroups. Proposition 3.16(b) shows that, if the Abelian group $G$ is the trajectory of a finitely generated $\phi$-inert subgroup $H$, then $\operatorname{ent}(\phi)=\widetilde{\operatorname{ent}}(\phi, H)$. On the other hand, it is possible to find examples where $G$ is the trajectory of a finitely generated subgroup, but it is not possible to find a finite set of elements generating a $\phi$-inert subgroup (take for example the right Bernoulli shift on the group $\left.\mathbb{Z}^{(\mathbb{N})}\right)$. We will prove in the following proposition that this inconvenience may occur only if the torsion-free rank of $G$ is infinite.

Proposition 5.6. Let $G$ be an Abelian group, $\phi: G \rightarrow G$ an endomorphism and suppose that there exists a finitely generated subgroup $F$ of $G$ such that $G=T(\phi, F)$. Then the following conditions are equivalent:

(a) $\widetilde{\operatorname{ent}}(\phi)<\infty$;

(b) $\operatorname{rk}(G)<\infty$;

(c) there exists $n \in \mathbb{N}_{+}$such that $T_{n}(\phi, F)$ is $\phi$-inert.

Furthermore, if the above equivalent conditions are not verified, then $G$ has a $\phi$-inert subgroup isomorphic to $\mathbb{Z}^{(\mathbb{N})}$ on which the restriction of $\phi$ acts as the right Bernoulli shift $\beta_{\mathbb{Z}}$.

Proof. (a) $\Rightarrow$ (b) Suppose $\operatorname{rk}(G)=\infty$. Let $x_{1}, \ldots, x_{k}$ be a set of generators of $F$, in particular, $G=$ $T\left(\phi, x_{1} \mathbb{Z}\right)+\ldots+T\left(\phi, x_{k} \mathbb{Z}\right)$ and so $\operatorname{rk}(G) \leq \sum_{i=1}^{k} \operatorname{rk}\left(T\left(\phi, x_{i} \mathbb{Z}\right)\right)$. In particular, $\operatorname{rk}\left(T\left(\phi, x_{i} \mathbb{Z}\right)\right)=\infty$ for some $i$. But the condition that $\operatorname{rk}\left(T\left(\phi, x_{i} \mathbb{Z}\right)\right)=\infty$ is equivalent to the fact that $\phi^{n}\left(x_{i}\right) \mathbb{Z} \cong \mathbb{Z}$ and 
$T_{n}\left(\phi, x_{i}\right) \cong \mathbb{Z}^{n}$, for every $n \in \mathbb{N}_{+}$. This means exactly that $T\left(\phi, x_{i} \mathbb{Z}\right) \cong \mathbb{Z}^{(\mathbb{N})}$ and $\phi$ acts on this group as a right Bernoulli shift. From Example 3.7 we deduce that $\widetilde{\operatorname{ent}}(\phi)=\infty$.

(b) $\Rightarrow$ (c) There exists a finitely generated subgroup $F^{\prime}$ of $G$ such that $F^{\prime} \cong \mathbb{Z}^{k}$ and $G / F^{\prime}$ is torsion. Since $G=T(\phi, F)$ and $F^{\prime}$ is finitely generated, there exists $n \in \mathbb{N}_{+}$such that $F^{\prime} \subseteq T_{n}(\phi, F)$. Now $\left(T_{n}(\phi, F)+\phi\left(T_{n}(\phi, F)\right)\right) / T_{n}(\phi, F)$ is torsion, because it is contained in a quotient of $G / F^{\prime}$, and also finitely generated, hence it is finite, so $T_{n}(\phi, F)$ is $\phi$-inert as desired.

(c) $\Rightarrow$ (a) Since $T_{n}(\phi, F)$ is also finitely generated, by Proposition 3.16 (b) one can see that $\widetilde{\operatorname{ent}}(\phi)=$ $\widetilde{\operatorname{ent}}\left(\phi, T_{n}(\phi, F)\right)$, therefore,

$$
\widetilde{\operatorname{ent}}(\phi) \leq \log \left|T_{n+1}(\phi, F) / T_{n}(\phi, F)\right|<\infty,
$$

and this concludes the proof.

The following technical lemma is needed in the proof of Proposition 5.8

Lemma 5.7. Let $G$ be an Abelian group of finite rank, $\phi: G \rightarrow G$ an endomorphism and suppose there exists a finitely generated subgroup $F$ of $G$ such that $G=T(\phi, F)$. Then $t(G)$ is bounded, hence $G=t(G) \oplus K$, where $K$ is torsion-free. Hence $\phi$ can be represented in matrix form as follows $\left(\phi_{t} \in \operatorname{End}(t(G)), \phi^{*} \in \operatorname{Hom}(K, t(G)), \bar{\phi} \in \operatorname{End}(K)\right):$

$$
\left(\begin{array}{cc}
\phi_{t} & \phi^{*} \\
0 & \bar{\phi}
\end{array}\right): t(G) \oplus K \rightarrow t(G) \oplus K \quad\left(\begin{array}{c}
t \\
f
\end{array}\right) \mapsto\left(\begin{array}{c}
\phi_{t}(t)+\phi^{*}(f) \\
\bar{\phi}(f)
\end{array}\right) .
$$

Furthermore, there exist subgroups $H_{1}$ of $t(G)$ and $H_{2}$ of $K$ such that:

(i) $H_{1}$ is finite, it contains $\phi^{*}\left(T\left(\bar{\phi}, H_{2}\right)\right)$ and $T\left(\phi_{t}, H_{1}\right)=t(G)$;

(ii) $\mathrm{H}_{2}$ is finitely generated, $\bar{\phi}$-inert and $T\left(\bar{\phi}, H_{2}\right)=K$;

(iii) $H=H_{1} \oplus H_{2}$ is finitely generated, $\phi$-inert and $T(\phi, H)=G$;

(iv) $\widetilde{\operatorname{ent}}(\phi, H)=\widetilde{\operatorname{ent}}\left(\phi_{t}, H_{1}\right)+\widetilde{\operatorname{ent}}\left(\bar{\phi}, H_{2}\right)$.

Proof. By hypothesis $G_{\phi}$ is a finitely generated $\mathbb{Z}[X]$-module. As $\mathbb{Z}[X]$ is a Noetherian ring, the $\mathbb{Z}[X]$ submodule $T=t(G)_{\phi}$ is again finitely generated, so $T=T\left(\phi, F_{1}\right)$ for a finite subgroup $F_{1}$. A positive integer annihilating $F_{1}$ annihilates the whole $T$, which is therefore bounded, hence a summand of $G$.

Thus, we can adopt the notation of the statement and let $\pi: G \rightarrow K$ be the canonical projection. We have that $K=T(\bar{\phi}, \pi F)$ satisfies the hypothesis of Proposition 5.6 and so, there exists $n \geq 1$ such that $H_{2}=T_{n}(\bar{\phi}, \pi F)$ is a $\bar{\phi}$-inert subgroup of $K$. We let also $H_{1}=F_{1}+\phi^{*}\left(T\left(\bar{\phi}, H_{2}\right)\right)$.

(i) As $\phi^{*}\left(T\left(\bar{\phi}, H_{2}\right)\right)$ is bounded, there exists $m \geq 1$ such that $m \phi^{*}\left(T\left(\bar{\phi}, H_{2}\right)\right)=0$. In particular, $m T\left(\bar{\phi}, H_{2}\right) \subseteq \operatorname{ker}\left(\phi^{*}\right) \cap T\left(\bar{\phi}, H_{2}\right)$ and so $\phi^{*}\left(T\left(\bar{\phi}, H_{2}\right)\right)$ is a quotient of $T\left(\bar{\phi}, H_{2}\right) / m T\left(\bar{\phi}, H_{2}\right)$, which is a finite group, since $T\left(\bar{\phi}, H_{2}\right)$ has finite rank. Hence $H_{1}$ is the sum of two finite groups, thus it is finitely generated and $\phi_{t}$-inert. Furthermore, the inclusions $T=T\left(\phi_{t}, F_{1}\right) \subseteq T\left(\phi_{t}, H_{1}\right) \subseteq T$ show that $T\left(\phi_{t}, H_{1}\right)=T$.

(ii) easily follows by construction.

(iii) The subgroup $H$ is finitely generated as it is the sum of two finitely generated subgroups. Since $H_{2}$ is $\phi$-inert by part (ii), $H$ is $\phi$-inert by Lemma 2.6. Finally, the inclusion $T\left(\phi_{t}, H_{1}\right) \oplus T\left(\bar{\phi}, H_{2}\right) \subseteq$ $T(\phi, H)$ shows that $G=t(G) \oplus K=T(\phi, H)$.

(iv) We want now to compute the $n$-trajectories of $H$. It is useful here to use the matrix notation, with the obvious meaning of symbols. Indeed, we have:

$$
H=\left(\begin{array}{l}
H_{1} \\
H_{2}
\end{array}\right), \quad \phi(H) \subseteq\left(\begin{array}{c}
\phi_{t}\left(H_{1}\right)+\phi^{*}\left(H_{2}\right) \\
\bar{\phi}\left(H_{2}\right)
\end{array}\right), \quad \phi^{2}(H) \subseteq\left(\begin{array}{c}
\phi_{t}^{2}\left(H_{1}\right)+\phi_{t} \phi^{*}\left(H_{2}\right)+\phi^{*} \bar{\phi}\left(H_{2}\right) \\
\bar{\phi}^{2}\left(H_{2}\right)
\end{array}\right), \ldots
$$


Going on this way, one obtains the inclusion

$$
\phi^{n}(H) \subseteq\left(\begin{array}{c}
\phi_{t}^{n} H_{1}+\sum\left\{\phi_{t}^{i} \phi^{*} \bar{\phi}^{j}\left(H_{2}\right): i+j=n-1,0 \leq i, j \leq n-1\right\} \\
\bar{\phi}^{n}\left(H_{2}\right)
\end{array}\right)
$$

It is clear that $\sum \phi_{t}^{i} \phi^{*} \bar{\phi}^{j} H_{2} \subseteq \sum \phi_{t}^{i} \phi^{*}\left(T\left(\bar{\phi}, H_{2}\right)\right)$. Now recall that by construction, $\phi^{*}\left(T\left(\bar{\phi}, H_{2}\right)\right) \leq H_{1}$ and so we can improve the above inclusion as follows

$$
\phi^{n}(H) \subseteq\left(\begin{array}{c}
\phi_{t}^{n}\left(H_{1}\right)+\sum_{i=0}^{n-1} \phi_{t}^{i}\left(H_{1}\right) \\
\bar{\phi}^{n}\left(H_{2}\right)
\end{array}\right)
$$

An easy computation now gives

$$
T_{n}(\phi, H) \subseteq\left(\begin{array}{c}
T_{n}\left(\phi_{t}, H_{1}\right) \\
T_{n}\left(\bar{\phi}, H_{2}\right)
\end{array}\right)
$$

Hence we obtain that $T_{n}(\phi, H) / H \subseteq T_{n}\left(\phi_{t}, H_{1}\right) / H_{1} \oplus T_{n}\left(\bar{\phi}, H_{2}\right) / H_{2}$. This shows that

$$
\widetilde{\operatorname{ent}}(\phi, H) \leq \widetilde{\operatorname{ent}}\left(\phi_{t}, H_{1}\right)+\widetilde{\operatorname{ent}}\left(\bar{\phi}, H_{2}\right) \text {. }
$$

Since the other inequality holds by Lemma 3.10(b), we get the wanted equality.

The next result proves the Addition Theorem with respect to the torsion subgroup.

Proposition 5.8. Let $G$ be an Abelian group, $\phi: G \rightarrow G$ an endomorphism and $\bar{\phi}: G / t(G) \rightarrow G / t(G)$ the endomorphism induced by $\phi$. Suppose there exists a finitely generated subgroup $F$ of $G$ such that $G=T(\phi, F)$. Then $\widetilde{\operatorname{ent}}(\phi)=\widetilde{\operatorname{ent}}\left(\phi \Gamma_{t(G)}\right)+\widetilde{\operatorname{ent}}(\bar{\phi})$.

Proof. Suppose that $\operatorname{rk}(G)$ is infinite. By Proposition $5.6 \widetilde{\operatorname{ent}}(\phi)=\infty$ and $G / t(G)$ has a $\phi$-invariant subgroup isomorphic to $\mathbb{Z}^{(\mathbb{N})}$ on which $\bar{\phi}$ acts as a right Bernoulli shift, so clearly $\widetilde{\operatorname{ent}}(\phi)=\infty=\widetilde{\operatorname{ent}}(\bar{\phi})$. So assume that $\operatorname{rk}(G)$ is finite. By Lemma $5.7 t(G)$ splits in $G$ and so $G=t(G) \oplus K$, where $K$ is torsionfree. Again by Lemma 5.7 there exist a finite subgroup $H_{1}$ of $t(G)$ and a finitely generated subgroup $H_{2}$ of $K$ such that $T\left(\phi \uparrow_{t(G)}, H_{1}\right)=t(G)$ and $T\left(\bar{\phi}, H_{2}\right)=K$; moreover $H=H_{1} \oplus H_{2}$ is finitely generated, $\phi$-inert, $T(\phi, H)=G$ and $\widetilde{\operatorname{ent}}(\phi, H)=\widetilde{\operatorname{ent}}\left(\phi_{t}, H_{1}\right)+\widetilde{\operatorname{ent}}\left(\bar{\phi}, H_{2}\right)$. In order to conclude, notice that $\widetilde{\operatorname{ent}}(\phi, H)=\widetilde{\operatorname{ent}}(\phi), \widetilde{\operatorname{ent}}\left(\phi \uparrow_{t(G)}, H_{1}\right)=\widetilde{\operatorname{ent}}\left(\phi_{t}\right)$ and $\widetilde{\operatorname{ent}}\left(\bar{\phi}, H_{2}\right)=\widetilde{\operatorname{ent}}(\bar{\phi})$ by Proposition 3.16(b).

The preceding results allow us to prove the Addition Theorem for the intrinsic algebraic entropy in case the Abelian group we are dealing with is the trajectory of a finitely generated subgroup. The general case will follow using Proposition 5.2 .

Theorem 5.9. Let $G$ be an Abelian group, $\phi$ an endomorphism and suppose that there exists a finitely generated subgroup $F$ of $G$ such that $G=T(\phi, F)$. Then, for every $\phi$-invariant subgroup $H$ of $G$ we have that

$$
\widetilde{\operatorname{ent}}(\phi)=\widetilde{\operatorname{ent}}\left(\phi \uparrow_{H}\right)+\widetilde{\operatorname{ent}}(\bar{\phi}),
$$

where $\bar{\phi}$ denotes the endomorphism induced by $\phi$ on the quotient $G / H$.

Proof. Obviously, $\operatorname{rk}(G)=\infty$ if and only if $\operatorname{rk}(H)=\infty$ or $\operatorname{rk}(G / H)=\infty, \operatorname{as} \operatorname{rk}(G)=\operatorname{rk}(H)+\operatorname{rk}(G / H)$. By Proposition 5.6, this means that $\widetilde{\operatorname{ent}}(\phi)=\infty$ if and only if $\widetilde{\operatorname{ent}}\left(\phi \uparrow_{H}\right)=\infty \operatorname{or} \widetilde{\operatorname{ent}}(\bar{\phi})=\infty$. This allows us to suppose, as we will do from now on, that $G$ has finite rank. In order to simplify the notation, we will adopt the following conventions. All the groups that will appear in this proof will be of 
the form $K_{1} / K_{2}$, with $K_{2} \leq K_{1} \phi$-invariant subgroups of $G$, so that $\phi$ induces an endomorphism $\phi_{K_{1} / K_{2}}$ of $K_{1} / K_{2}$. We will denote $\widetilde{\operatorname{ent}}\left(\phi_{K_{1} / K_{2}}\right)$ simply by $\widetilde{\operatorname{ent}}\left(K_{1} / K_{2}\right)$. Furthermore, we let $K=t(G)+H$.

Let us list some short exact sequences of $\mathbb{Z}[X]$-modules on which ent is additive (recall that we are assuming $G$ of finite rank).

(1) From $0 \rightarrow t(G) \rightarrow G \rightarrow G / t(G) \rightarrow 0$ we have that $\widetilde{\operatorname{ent}}(G)=\widetilde{\operatorname{ent}}(t(G))+\widetilde{\operatorname{ent}}(G / t(G))$ by Proposition 5.8 .

(2) From $0 \rightarrow t(H) \rightarrow H \rightarrow K / t(G) \rightarrow 0$ we have that $\widetilde{\operatorname{ent}}(H)=\widetilde{\operatorname{ent}}(t(H))+\widetilde{\operatorname{ent}}(K / t(G))$ by Proposition 5.8 .

(3) From $0 \rightarrow t(H) \rightarrow t(G) \rightarrow t(G) / t(H) \rightarrow 0$ we have that $\widetilde{\operatorname{ent}}(t(G))=\widetilde{\operatorname{ent}}(t(H))+\widetilde{\operatorname{ent}}(t(G) / t(H))$ by the Addition Theorem for ent (see [9]) and the fact that ent $=$ ent on torsion Abelian groups.

(4) Considering the short exact sequence in (1.6) (replacing there $G$ by $G / t(G)$ and $L$ by $K / t(G)$ ), and noting that $(G / t(G)) /(K / t(G)) \cong G / K$, we obtain the short exact sequence:

$$
0 \rightarrow(K / t(G))^{*} \rightarrow G / t(G) \rightarrow \frac{G / K}{t(G / K)} \rightarrow 0 .
$$

So we have that $\widetilde{\operatorname{ent}}(G / t(G))=\widetilde{\operatorname{ent}}\left((K / t(G))^{*}\right)+\widetilde{\operatorname{ent}}((G / K) / t(G / K))$ by Corollary 5.4 .

(5) From $0 \rightarrow K / t(G) \rightarrow(K / t(G))^{*} \rightarrow(K / t(G))^{*} /(K / t(G)) \rightarrow 0$ we have that $\widetilde{\operatorname{ent}}(K / t(G))=$ $\widetilde{\operatorname{ent}}\left((K / t(G))^{*}\right)$, by Proposition 5.5 .

(6) It is not difficult to verify that $(G / K) / t(G / K) \cong(G / H) / t(G / H)$ (to see this just notice that the two canonical surjections $G \rightarrow(G / K) / t(G / K)$ and $G \rightarrow(G / H) / t(G / H)$ have the same kernel), and so $\widetilde{\operatorname{ent}}((G / K) / t(G / K))=\widetilde{\operatorname{ent}}((G / H) / t(G / H))$.

(7) From $0 \rightarrow t(G / H) \rightarrow G / H \rightarrow(G / H) / t(G / H) \rightarrow 0$ we have, again by Proposition 5.8, that $\widetilde{\operatorname{ent}}(G / H)=\widetilde{\operatorname{ent}}(t(G / H))+\widetilde{\operatorname{ent}}((G / H) / t(G / H))$.

(8) The quotient $K / H$ is contained in $t(G / H)$ and it is the kernel of the projection $t(G / H) \rightarrow$ $t(G / K)$. Moreover, $K / H \cong t(G) / t(H)$. So we consider the short exact sequence of torsion Abelian groups

$$
0 \rightarrow t(G) / t(H) \rightarrow t(G / H) \rightarrow t(G / K) \rightarrow 0 .
$$

Since

$$
t(G / K) \cong t\left(\frac{G / t(G)}{K / t(G)}\right)=\frac{(K / t(G))^{*}}{K / t(G)},
$$

and the latter quotient is finite by Proposition 5.5, we can conclude that $t(G / K)$ is finite. So $\widetilde{\operatorname{ent}}(t(G / K))=0$. By the Addition Theorem for ent (see [9]) and the fact that ent $=$ ent on torsion Abelian groups, we have $\widetilde{\operatorname{ent}}(t(G / H))=\widetilde{\operatorname{ent}}(t(G) / t(H))$.

Now we can conclude the proof with the following series of equalities:

$$
\begin{array}{rlr}
\widetilde{\operatorname{ent}}(G) & =\widetilde{\operatorname{ent}}(t(G))+\widetilde{\operatorname{ent}}(G / t(G)) & \text { by (1) } \\
& =\widetilde{\operatorname{ent}}(t(H))+\widetilde{\operatorname{ent}}(t(G) / t(H))+\widetilde{\operatorname{ent}}\left((K / t(G))^{*}\right)+\widetilde{\operatorname{ent}}((G / K) / t(G / K)) & \text { by (3) and (4) } \\
& =\widetilde{\operatorname{ent}}(t(H))+\widetilde{\operatorname{ent}}(t(G) / t(H))+\widetilde{\operatorname{ent}}(K / t(G))+\widetilde{\operatorname{ent}}((G / H) / t(G / H)) & \text { by (5) and (6) } \\
& =\widetilde{\operatorname{ent}}(H)+\widetilde{\operatorname{ent}}(t(G) / t(H))+\widetilde{\operatorname{ent}}(G / H)-\widetilde{\operatorname{ent}}(t(G / H)) & \text { by (2) and (7) } \\
& =\widetilde{\operatorname{ent}}(H)+\widetilde{\operatorname{ent}}(G / H) & \text { by (8). }
\end{array}
$$

This concludes the proof. 
Corollary $\mathbf{5 . 1 0}$ (Addition Theorem). The intrinsic algebraic entropy $\widetilde{\text { ent }}: \operatorname{Mod}(\mathbb{Z}[X]) \rightarrow \mathbb{R}^{*}$ is an additive invariant, hence it is a length function.

Proof. In Lemma 3.14 we proved that ent is an upper continuous invariant, so it is a length function provided that it is additive. The above Theorem 5.9 shows that ent is additive on the class of finitely generated $\mathbb{Z}[X]$-modules. Since $\mathbb{Z}[X]$ is Noetherian, Proposition 5.2 applies to show that ent is additive on the whole category $\operatorname{Mod}(\mathbb{Z}[X])$.

We can easily derive from the Addition Theorem a characterization of the endomorphisms having intrinsic algebraic entropy zero.

Corollary 5.11. Let $G$ be an Abelian group and $\phi: G \rightarrow G$ an endomorphism. Then $\widetilde{\operatorname{ent}}(\phi)=0$ if and only if the following conditions are satisfied:

(a) $\operatorname{ent}\left(\phi \uparrow_{t(G)}\right)=0$;

(b) $G$ is the union of a chain of pure $\phi$-invariant subgroups

$$
t(G)=G_{0}<G_{1}<G_{2}<\ldots<G_{\sigma}<\ldots<\bigcup_{\sigma<\lambda} G_{\sigma}=G
$$

such that $\operatorname{rk}\left(G_{\sigma+1} / G_{\sigma}\right)$ is finite for all $\sigma$ and, denoting by $\phi_{\sigma+1}: G_{\sigma+1} / G_{\sigma} \rightarrow G_{\sigma+1} / G_{\sigma}$ the endomorphism induced by $\phi, G_{\sigma+1} / G_{\sigma}$ is the pure closure in $G / G_{\sigma}$ of a cyclic $\phi_{\sigma+1}$-trajectory for all $\sigma<\lambda$;

(c) the unique extension $\widetilde{\phi}_{\sigma+1}$ of $\phi_{\sigma+1}$ to the divisible hull of $G_{\sigma+1} / G_{\sigma}$ has monic characteristic polynomial over $\mathbb{Z}$.

Proof. First assume that (a), (b) and (c) are true. We prove that $\widetilde{\operatorname{ent}}\left(\phi \uparrow_{G_{\sigma}}\right)=0$ for each ordinal $\sigma$, by transfinite induction on $\sigma$; the fact that $\widetilde{\operatorname{ent}}(\phi)=0$ then follows by Lemma 5.1. Item (a) provides the initial step for $\sigma=0$. Assume $\widetilde{\operatorname{ent}}\left(\phi\left\lceil_{G_{\sigma}}\right)=0\right.$ and consider the exact sequence:

$$
0 \rightarrow G_{\sigma} \rightarrow G_{\sigma+1} \rightarrow G_{\sigma+1} / G_{\sigma} \rightarrow 0 .
$$

The endomorphism $\phi_{\sigma+1}$ of $G_{\sigma+1} / G_{\sigma}$ induced by $\phi\left\lceil_{G_{\sigma+1}}\right.$ has zero intrinsic algebraic entropy by (c), Corollary 3.15 and Theorem 4.2 then the Addition Theorem ensures that $\widetilde{\operatorname{ent}}\left(\phi \uparrow_{G_{\sigma+1}}\right)=0$. Assume now that $\sigma$ is a limit ordinal, and that $\widetilde{\operatorname{ent}}\left(\phi \uparrow_{G_{\rho}}\right)=0$ for all ordinals $\rho<\sigma$. Then Lemma 5.1 ensures that also $\widetilde{\operatorname{ent}}\left(\phi\left\lceil G_{\sigma}\right)=0\right.$, so we are done.

Conversely, let us assume that $\widetilde{\operatorname{ent}}(\phi)=0$. Then (a) follows from Lemma 3.10(a). The construction of the chain in (b) is made by transfinite induction. If $G_{\sigma}$ is already constructed, let $\psi_{\sigma}: G / G_{\sigma} \rightarrow$ $G / G_{\sigma}$ the map induced by $\phi$. Choose $x \in G \backslash G_{\sigma}$ and consider the $\psi_{\sigma}$-trajectory $T\left(\psi_{\sigma}, x+G_{\sigma}\right)=T_{\sigma}$ in $G / G_{\sigma}$; let $G_{\sigma+1} / G_{\sigma}=T_{\sigma}^{*}$ be its pure closure in $G / G_{\sigma}$. Finally, let $\phi_{\sigma+1}: G_{\sigma+1} / G_{\sigma} \rightarrow G_{\sigma+1} / G_{\sigma}$ be the restriction of $\psi_{\sigma}$ to $G_{\sigma+1} / G_{\sigma}$. If $\sigma$ is a limit ordinal, set $G_{\sigma}=\bigcup_{\rho<\sigma} G_{\rho}$. Then $\widetilde{\operatorname{ent}}(\phi)=0$ implies $\widetilde{\operatorname{ent}}\left(\psi_{\sigma+1}\right)=0$ for all $\sigma$, and consequently also that $\widetilde{\text { ent }}\left(\phi_{\sigma+1}\right)=0$ for all $\sigma$; this last equality has the consequence that $\operatorname{rk}\left(G_{\sigma+1} / G_{\sigma}\right)$ is finite, because $T_{\sigma}=T\left(\psi_{\sigma}, x+G_{\sigma}\right)$ of infinite rank would imply that $\widetilde{\operatorname{ent}}\left(\phi_{\sigma+1}\right)=\infty$, by Example 3.7 . Now item (c) follows from Corollary 3.15 and Theorem 4.2

As done in the case of the algebraic entropy in [9], it is possible to define the global intrinsic algebraic entropy of an Abelian group $G$ as

$$
\widetilde{\operatorname{ent}}(G)=\sup \{\widetilde{\operatorname{ent}}(\phi): \phi: G \rightarrow G \text { endomorphism }\} .
$$

The following is an example of a torsion-free Abelian group with $\operatorname{rk}(G)=2^{\aleph_{0}}$ and $\widetilde{\operatorname{ent}}(G)=0$. 
Example 5.12. It is well known that there exist $2^{\aleph_{0}}$ subgroups $R_{\sigma}$, with $\sigma<2^{\aleph_{0}}$, of the group $\mathbb{Q}$ of the rational numbers such that $\operatorname{Hom}_{\mathbb{Z}}\left(R_{\sigma}, R_{\tau}\right)=0$ if $\sigma \neq \tau$ and $\operatorname{End}\left(R_{\sigma}\right)=\mathbb{Z}$ for all $\sigma$ (for instance, take $2^{\aleph_{0}}$ infinite subsets $\sigma$ of $\mathbb{P}$ with finite mutual intersection, and for each $\sigma$ let $R_{\sigma}=\langle 1 / p: p \in \sigma\rangle$ ).

Let $G=\bigoplus_{\sigma} R_{\sigma}$, that clearly has $\operatorname{rk}(G)=2^{\aleph_{0}}$. Then $\operatorname{End}(G)=\prod_{\sigma} \mathbb{Z}$, and for every endomorphism $\phi$ of $G$ each subgroup $R_{\sigma}$ is $\phi$-invariant. Therefore $\phi: G \rightarrow G$ satisfies the conditions of Corollary 5.11, with $G_{\sigma}=\bigoplus_{\alpha \leq \sigma} R_{\alpha}$, so $\widetilde{\operatorname{ent}}(\phi)=0$. Hence $\widetilde{\operatorname{ent}}(G)=0$.

In the case of torsion Abelian groups $G$, Proposition 3.6 gives $\operatorname{ent}(G)=\widetilde{\operatorname{ent}}(G)$. Many examples of torsion Abelian groups $G$ with $\operatorname{ent}(G)=0$ can be found in [9]. Nevertheless, the problem of the classification of all torsion Abelian groups with this property is open (see [9, 19]).

\section{The Uniqueness Theorem}

In this section $h^{*}: \operatorname{Mod}(\mathbb{Z}[X]) \rightarrow \mathbb{R}^{*}$ denotes a length function and, given $G_{\phi} \in \operatorname{Mod}(\mathbb{Z}[X])$, we will often write $h^{*}(\phi)$ in place of $h^{*}\left(G_{\phi}\right)$. We characterize ent as the unique length function of $\operatorname{Mod}(\mathbb{Z}[X])$ satisfying two natural hypotheses. Indeed, we prove the following

Theorem 6.1 (Uniqueness Theorem). There exists a unique length function $h^{*}: \operatorname{Mod}(\mathbb{Z}[X]) \rightarrow \mathbb{R}^{*}$ such that

(a) $h^{*}\left(\beta_{K}\right)=\log |K|$ for any finite Abelian group $K$, where $\beta_{K}: K^{(\mathbb{N})} \rightarrow K^{(\mathbb{N})}$ is the right Bernoulli shift;

(b) $h^{*}(\phi)=\log s$ for every automorphism $\phi$ of $\mathbb{Q}^{m}$ whose characteristic polynomial over $\mathbb{Q}$ has the positive integer $s$ as least common multiple of the denominators of its coefficients.

Furthermore, $h^{*}=\widetilde{\text { ent. }}$.

The proof of the Uniqueness Theorem will follow at the end of this section after three lemmas describing some properties of the length functions of $\operatorname{Mod}(\mathbb{Z}[X])$. Note that an analogous result has been proved for the algebraic entropy $h$ in [6], with hypothesis (b) replaced by the Algebraic Yuzvinski Formula:

$\left(\mathrm{b}^{\prime}\right) h(\phi)=\mathrm{m}(\phi)$ for every automorphism $\phi$ of $\mathbb{Q}^{m}$ whose minimal polynomial over $\mathbb{Q}$ has the positive integer $s$ as least common multiple of the denominators of its coefficients.

Furthermore, it was proved in [9] that ent is the unique length function defined on the endomorphisms of torsion Abelian groups satisfying hypothesis (a). We remark that in [9] also the logarithmic law appeared in the hypothesis of such theorem, anyway one can use the following Lemma 6.2 to see that this is not necessary.

The next lemma proves that condition (a) in the statement of the Uniqueness Theorem implies that $h^{*}$ is trivial on all the endomorphisms of finite Abelian groups. The argument used in the proof already appeared in [19].

Lemma 6.2. Let $h^{*}: \operatorname{Mod}(\mathbb{Z}[X]) \rightarrow \mathbb{R}^{*}$ be a length function satisfying the hypothesis (a) of the Uniqueness Theorem. Then $h^{*}(\phi)=0$ for all $\phi: G \rightarrow G$, with $G$ a finite Abelian group.

Proof. Consider the surjective homomorphism $f: G^{(\mathbb{N})} \rightarrow G$ such that $f\left(\left(x_{n}\right)_{n \in \mathbb{N}}\right)=\sum_{n \in \mathbb{N}} \phi^{n}\left(x_{n}\right)$; note also that $f \beta_{G}=\phi f$, and so $f$ is a surjective homomorphism of $\mathbb{Z}[X]$-modules $f: G_{\beta_{G}}^{(\mathbb{N})} \rightarrow G_{\phi}$. Thus, by the additivity of $h^{*}$,

$$
h^{*}(\phi)=h^{*}\left(\beta_{G}\right)-h^{*}\left(\operatorname{ker}(f)_{\beta_{G}}\right)
$$


On the other hand, consider the injective homomorphism $g: G^{(\mathbb{N})} \rightarrow G^{(\mathbb{N})}$ defined on the generators as follows

$$
g(0, \ldots, 0, \underbrace{x}_{n}, 0, \ldots)=(0, \ldots, 0, \underbrace{\phi(x)}_{n},-x, 0, \ldots) .
$$

Note that $g \beta_{G}=\beta_{G} g$ and $f g=0$; hence $g$ is an embedding of $\mathbb{Z}[X]$-modules of $G_{\beta_{G}}^{(\mathbb{N})}$ in $\operatorname{ker}(f)_{\beta_{G}}$ and so $h^{*}\left(\beta_{G}\right) \leq h^{*}\left(\operatorname{ker}(f)_{\beta_{G}}\right) \leq h^{*}\left(\beta_{G}\right)$. By (6.1), $h^{*}(\phi)=0$.

In the next lemma we prove that any length function $h^{*}$ of $\operatorname{Mod}(\mathbb{Z}[X])$ which satisfies condition (a) in the statement of the Uniqueness Theorem, necessarily coincides with ent on finitely generated $\mathbb{Z}[X]$-modules $G_{\phi}$ such that $\operatorname{rk}(G)=\infty$ (see also Proposition 5.6).

Lemma 6.3. Let $h^{*}: \operatorname{Mod}(\mathbb{Z}[X]) \rightarrow \mathbb{R}^{*}$ be a length function satisfying the hypothesis (a) of the Uniqueness Theorem. Let $G$ be an Abelian group of infinite rank, $\phi: G \rightarrow G$ an endomorphism and $F$ a finitely generated subgroup of $G$ such that $G=T(\phi, F)$. Then $h^{*}(\phi)=\infty$.

Proof. By Proposition 5.6 there is a $\phi$-invariant subgroup $H \cong \mathbb{Z}^{(\mathbb{N})}$ of $G$ on which the restriction of $\phi$ acts as the right Bernoulli shift $\beta_{\mathbb{Z}}$. Thus, it is enough to show that $h^{*}\left(H_{\phi}\right)=\infty$. This follows by the fact that $(H / p H)_{\phi} \cong \mathbb{Z}(p)_{\beta_{\mathbb{Z}(p)}}^{(\mathbb{N})}$ and so $h^{*}\left(H_{\phi}\right) \geq \sup \left\{h^{*}\left(\beta_{\mathbb{Z}(p)}\right): p \in \mathbb{P}\right\}=\sup \{\log (p): p \in \mathbb{P}\}=\infty$.

The next lemma is the last result that we need for the proof of the Uniqueness Theorem, the proof is exactly the same of Corollary 3.15 and so it is omitted.

Lemma 6.4. Let $h^{*}: \operatorname{Mod}(\mathbb{Z}[X]) \rightarrow \mathbb{R}^{*}$ be an upper continuous invariant. Let $G$ be a torsion-free Abelian group and $\phi: G \rightarrow G$ an endomorphism. Then $h^{*}(\phi)=h^{*}(\widetilde{\phi})$, where $\widetilde{\phi}: D(G) \rightarrow D(G)$ is the unique extension of $\phi$ to the divisible hull $D(G)$ of $G$.

Proof of the Uniqueness Theorem 6.1. We already noted that $\widetilde{\text { ent }}$ is an additive upper continuous invariant; it satisfies (a) since over torsion Abelian groups it coincides with ent by Proposition 3.6 and because of [9, Example 1.9]; finally, it satisfies (b) in view of Theorem 4.2.

Conversely, let $h^{*}$ be a length function on $\operatorname{Mod}(\mathbb{Z}[X])$ satisfying (a) and (b). We have to show that $h^{*}$ coincides with ent. It suffices to verify that these two length functions coincide on finitely generated $\mathbb{Z}[X]$-modules. So assume without loss of generality that $G=T(\phi, F)$ for some finitely generated subgroup $F$ of $G$.

If $G$ is torsion, then $G$ is either finite or there exists an element $x \in G[p]$ for some prime $p$ such that $T(\phi, x \mathbb{Z}) \cong \mathbb{Z}(p)^{(\mathbb{N})}$ and $\phi$ acts on this subgroup as the right Bernoulli shift $\beta_{\mathbb{Z}(p)}$. So, using an inductive argument, one can realize the group $G$ as the union of a finite chain of $\phi$-invariant subgroups

$$
0=\left(H_{0}\right)_{\phi} \subseteq\left(H_{1}\right)_{\phi} \subseteq \cdots \subseteq\left(H_{n}\right)_{\phi}=G_{\phi}
$$

such that $\left(H_{i+1} / H_{i}\right)_{\phi}$ is either finite or conjugated to $\mathbb{Z}\left(p_{i}\right)_{\beta_{\mathbb{Z}\left(p_{i}\right)}^{(\mathbb{N})}}$, for all $i<n$ and suitable primes $p_{i}$. Hence, $\widetilde{\operatorname{ent}}(\phi)=h^{*}(\phi)$ by the additivity of both invariants, condition (a) and Lemma 6.2 .

If $G$ is torsion-free, then $\widetilde{\operatorname{ent}}(\phi)=h^{*}(\phi)$ by (b) and Lemma 6.4 in case $\operatorname{rk}(G)<\infty$, by Lemma 6.3 and Proposition 5.6 in case $\operatorname{rk}(G)=\infty$.

The general case follows by the torsion and torsion-free cases using additivity.

\section{Acknowledgements}

We are indebted to the referee for her/his careful criticisms and useful comments. 


\section{References}

[1] D. Arnold, Finite Rank Torsion Free Abelian Groups and Rings, L.N.M. n. 931, Springer, 1982.

[2] R. L. Adler, A. G. Konheim, M. H. McAndrew, Topological entropy, Trans. Amer. Math. Soc. 114 (1965), 309-319.

[3] V.V. Belyaev, Inert subgroups in infinite simple groups, Siberian Math. J. 34 (1993), 218-232.

[4] V.V. Belyaev, M. Kuzucuoğlu, E. Seçkin, Totally inert groups, Rend. Sem. Mat. Univ. Padova 102 (1999), 151-156.

[5] U. Dardano, S. Rinauro, Inertial automorphisms of an Abelian group, Rend. Sem. Mat. Univ. Padova 127 (2012), 213-233.

[6] D. Dikranjan, A. Giordano Bruno, Entropy on Abelian groups, preprint, http://arxiv.org/abs/1007.0533.

[7] D. Dikranjan, A. Giordano Bruno, Topological entropy and algebraic entropy for group endomorphisms, Arhangelskiu A. V., Moiz ud Din Khan; Kocinac L., ed., Proceedings Islamabad ICTA 2011, Cambridge Scientific Publishers 133-214.

[8] D. Dikranjan, A. Giordano Bruno, L. Salce, S. Virili, Fully inert subgroups of divisible Abelian groups, J. Group Theory 16 (2013), 915-939.

[9] D. Dikranjan, B. Goldsmith, L. Salce, P. Zanardo, Algebraic entropy of endomorphisms of Abelian groups, Trans. Amer. Math. Soc. 361 (2009), 3401-3434.

[10] D. Dikranjan, L. Salce, P. Zanardo, Fully inert subgroups of free Abelian groups, to appear in Periodica Math. Hungarica.

[11] D. Dikranjan, M. Sanchis, S. Virili, New and old facts about entropy on uniform spaces and topological groups, Topol. Appl. 159 (2012), 1916-1942.

[12] A. Giordano Bruno, S. Virili, The Algebraic Yuzvinski Formula, to appear in J. Algebra.

[13] D. H. Lehmer, Factorization of certain cyclotomic functions, Ann. of Math. 34 (1933), 461-469.

[14] M. J. Mossinghoff, G. Rhin, Q. Wu, Minimal Mahler measures, Experiment. Math. 17 (4) (2008), 451-458.

[15] D. G. Northcott and M. Reufel, A generalization of the concept of lenght, Quart. J. of Math. (Oxford) (2) 16 (1965), 297-321.

[16] J. Peters, Entropy on discrete Abelian groups, Adv. Math. 33 (1979), 1-13.

[17] J. Peters, Entropy of automorphisms on L.C.A. groups, Pacific J. Math. 96 (2) (1981), 475-488.

[18] D. J. S. Robinson, On inert subgroups of a group, Rend. Sem. Mat. Univ. Padova 115 (2006), $137-159$.

[19] L. Salce, Some results on the algebraic entropy, Proc. 2011 Muelheim Conference, Contemporary Math. AMS 576 (2012), 297-304. 
[20] L. Salce, P. Vamos, S. Virili, Length functions, multiplicities and algebraic entropy, Forum Math. 25(2) (2013), 255-282.

[21] P. Vámos, Length Functions on Modules, Ph.D. Thesis, University of Sheffield, 1968.

[22] P. Vámos, Additive Functions and Duality over Noetherian Rings, Quart. J. of Math. (Oxford) (2) 19 (1968), 43-55.

[23] D. van Dantzig, Studien over topologische Algebra, Dissertation, Amsterdam 1931.

[24] S. Virili, Entropy for endomorphisms of LCA groups, Topol. Appl. 159 (9) (2012), 2546-2556.

[25] M. D. Weiss, Algebraic and other entropies of group endomorphisms, Math. Systems Theory 8 (3) $(1974 / 75), 243-248$.

[26] G. A. Willis, The structure of totally disconnected, locally compact groups, Math. Ann. 300 (2) (1994), 341-363.

[27] G. A. Willis, Further properties of the scale function on a totally disconnected group, J. Algebra 237 (1) (2001), 142-164.

Addresses of the authors:

Dikran Dikranjan - dikran.dikranjan@uniud.it

Anna Giordano Bruno - anna.giordanobruno@uniud.it

Dipartimento di Matematica e Informatica, Università di Udine

Via delle Scienze 206 - 33100 Udine, Italy

Luigi Salce - salce@math.unipd.it

Dipartimento di Matematica, Università di Padova

Via Trieste 63 - 35121 Padova, Italy

Simone Virili - simone@mat.uab.cat

Departament de Matemàtiques, Universitat Autònoma de Barcelona

Edifici C - 08193 Bellaterra (Barcelona), Spain. 